ACCEPTED MANUSCRIPT

\title{
Osteogenic and angiogenic tissue formation in high fidelity nanocomposite Laponite-gelatin bioinks
}

To cite this article before publication: Gianluca Cidonio et al 2019 Biofabrication in press https://doi.org/10.1088/1758-5090/ab19fd

\section{Manuscript version: Accepted Manuscript}

Accepted Manuscript is "the version of the article accepted for publication including all changes made as a result of the peer review process, and which may also include the addition to the article by IOP Publishing of a header, an article ID, a cover sheet and/or an 'Accepted Manuscript' watermark, but excluding any other editing, typesetting or other changes made by IOP Publishing and/or its licensors"

This Accepted Manuscript is @ 2018 IOP Publishing Ltd.

During the embargo period (the 12 month period from the publication of the Version of Record of this article), the Accepted Manuscript is fully protected by copyright and cannot be reused or reposted elsewhere.

As the Version of Record of this article is going to be / has been published on a subscription basis, this Accepted Manuscript is available for reuse under a CC BY-NC-ND 3.0 licence after the 12 month embargo period.

After the embargo period, everyone is permitted to use copy and redistribute this article for non-commercial purposes only, provided that they adhere to all the terms of the licence https://creativecommons.org/licences/by-nc-nd/3.0

Although reasonable endeavours have been taken to obtain all necessary permissions from third parties to include their copyrighted content within this article, their full citation and copyright line may not be present in this Accepted Manuscript version. Before using any content from this article, please refer to the Version of Record on IOPscience once published for full citation and copyright details, as permissions will likely be required. All third party content is fully copyright protected, unless specifically stated otherwise in the figure caption in the Version of Record.

View the article online for updates and enhancements. 


\title{
Osteogenic and angiogenic tissue formation in high fidelity nanocomposite Laponite-gelatin bioinks
}

\author{
Gianluca Cidonio ${ }^{1,2^{*}}$ \& Cesar R. Alcala-Orozco ${ }^{3 *}$, Khoon S. Lim ${ }^{3}$,Michael \\ Glinka $^{1}$, Isha Mutreja ${ }^{3}$, Yang-Hee Kim ${ }^{1}$, Jonathan I. Dawson ${ }^{1}$, Tim B.F.
} Woodfield $^{3^{* *}}$ \& Richard O.C. Oreffo ${ }^{1^{* *}}$

* These authors contributed equally

** Authors to whom any correspondence should be addressed

${ }^{1}$ Bone and Joint Research Group, Centre for Human Development, Stem Cells and Regeneration, Institute of Developmental Sciences, University of Southampton, Southampton, United Kingdom

${ }^{2}$ Engineering Materials Research Group, Faculty of Engineering and the Environment, University of Southampton, Southampton, United Kingdom

${ }^{3}$ Christchurch Regenerative Medicine and Tissue Engineering (CReaTE) Group, Department of Orthopaedics Surgery and Musculoskeletal Medicine, University of Otago Christchurch, Christchurch, New Zealand

Email: richard.oreffo@soton.ac.uk; tim.woodfield@otago.ac.nz

Keywords: Laponite, GelMA, bioink, visible light, biofabrication, growth factor delivery, hydrogels, ex vivo 


\section{Abstract}

Bioprinting of living cells is rapidly developing as an advanced biofabrication approach to engineer tissues. Bioinks can be extruded in three-dimensions (3D) to fabricate complex and hierarchical constructs for implantation. However, lack of functionality can often be attributed to poor bioink properties. Indeed, advanced bioinks encapsulating living cells should: (i) present optimal rheological properties and retain 3D structure post-fabrication, (ii) promote cell viability and support cell differentiation, (iii) localise proteins of interest (e.g. vascular endothelial growth factor (VEGF)) to stimulate encapsulated cell activity and tissue ingrowth upon implantation. In this study, we present the results of the inclusion of a synthetic nanoclay, Laponite (LPN) together with a gelatin methacryloyl (GelMA) bioink and the development of a functional cellinstructive bioink. A nanocomposite bioink displaying enhanced shape fidelity retention and interconnected porosity within extrusion-bioprinted fibres was observed. Human bone marrow stromal cell (HBMSC) viability within the nanocomposite showed no significant changes over 21 days of culture in LPN-GelMA (85.60 $\pm 10.27 \%$ ), compared to a significant decrease in GelMA from $7(95.88 \pm 2.90 \%)$ to 21 days (55.54 \pm 14.72 \%) $(p<0.01)$. HBMSCs were observed to proliferate in LPN-GeIMA with a significant increase in cell number over 21 days ( $p<0.0001$ ) compared to GelMA alone. HBMSCsladen LPN-GelMA scaffolds supported osteogenic differentiation evidenced by mineralized nodule formation, including in the absence of the osteogenic drug dexamethasone. Ex vivo implantation in a chick chorioallantoic membrane (CAM) model, demonstrated excellent integration of the bioink constructs in the vascular chick embryo after 7 days of incubation. VEGF-loaded LPN-GelMA constructs demonstrated significantly higher vessel penetration than GelMA-VEGF $(p<0.0001)$ scaffolds. Integration and vascularisation was directly related to increased drug absorption and retention by LPN-GelMA compared to LPN-free GeIMA. In summary, a novel lightcurable nanocomposite bioink for 3D skeletal regeneration supportive of cell growth and growth factor retention and delivery, evidenced by ex vivo vasculogenesis, was developed with potential application in hard and soft tissue reparation. 


\section{Introduction}

Tissue engineering (TE) approaches over the last decades, have attempted to aid skeletal regeneration and while a significant body of evidence exists demonstrating excellent preclinical efficacy, limited success in clinical translation remains a challenge. Biofabrication technologies, including three-dimensional (3D) bioprinting and bioassembly, have come to the fore as a novel approach to address key TE limitations including the spatial control of cell-biomaterial-molecules in 3D matrices [1-3]. Bioprinting approaches typically require the use of bioinks, which are defined as a formulation of cells suitable for processing by an automated biofabrication technology that may also contain biologically active components and biomaterials [4]. Generally, bioinks must exhibit specific rheological properties (e.g. flowinitiation, shear thinning) to enable laminar extrusion of filaments and layer-by-layer stacking [5]. This concept has been previously presented as "shape fidelity" or the ability of a bioink to retain the printed structure and internal geometry immediately post fabrication $[5,6]$. Moreover, bioinks for skeletal applications should be able to preserve the viability of encapsulated living cells, promote cell function and localise cell-instructive cues to stimulate stem cell differentiation, tissue formation, encourage vascular network infiltration and allow host integration post-implantation.

Gelatin methacryloyl (GeIMA) is a photopolymerisable macromer [7] initially developed to provide a chemically stable hydrogel with the capacity to retain its physical shape at physiological temperature [8]. In recent times, GelMA hydrogels have been widely used as a cell-carrier given its potential to simulate the highly hydrated native 3D cellular microenvironment, support cell adhesion and aid functionality [9]. Nevertheless, extrusion-based bioprinting is often carried out at the expense of cell viability due to factors associated with the bioprinting process (e.g. shear stress, holding times) or the post-fabrication crosslinking processing (e.g. damage by UV-light) $[10,11]$. In particular, 
Lim et al. $[12,13]$ used ruthenium (Ru) and sodium persulfate (SPS) as an alternative visible-light photoinitiating system, to avoid UV damage and to improve the viability of encapsulated cells. The visible-light curing approach resulted in minimal oxygen inhibition during photocrosslinking, allowing the fabrication of scaffolds with enhanced shape fidelity in comparison to scaffolds fabricated using UV-light [14,15]

However, the use of GelMA as a bioink is limited by the low viscosity and the narrow biofabrication window afforded by this hydrogel, resulting in poor filament extrusion and shape fidelity over $20-37^{\circ} \mathrm{C}$ (room temperature to body temperature) [12,16], even at relatively high GelMA concentrations of $10-20$ wt\%. Encapsulated human bone marrow stromal cells (HBMSCs) targeted for osteogenic differentiation/ require a permissive hydrogel microenvironment to allow adequate cell function (i.e. cell spreading, cell communication and mechanosensing) [17,18], typically provided by low GelMA concentrations (5-10 wt\%). To date, development of approaches to overcome the narrow biofabrication window and shape retention of permissive hydrogels represents a major challenge in 3D bioprinting [19].

To address issues around printability, multiple GelMA composites have been developed through the blending of various materials including hyaluronic acid [16], gellan gum [20] and collagen [12] relating to their viscosity modifying and/or yield stress modifying capabilities. Nevertheless, none of the developed composite bioinks has targeted bioprinting of soft permissive hydrogels with high shape fidelity in combination with the skeletal-specific requirements of bone formation and blood vessel ingrowth [21].

Thus, while significant research has focused on adequate printability and high cell viability as the basic requirements for bioinks, advanced bioinks can be tailored to the target tissue through the incorporation of multi-functional fillers $[22,23]$. In the context of bone, the ideal bioink filler would provide enhanced printability and concomitantly grant cell-instructive properties towards bone formation and vascular growth. 
Clays have a long history of use in medical applications [24,25] and, in recent years have garnered significant interest as a biomaterial for regenerative medicine applications [26]. In particular, Laponite (LPN) is a smectite nanomaterial able to generate colloidal-like suspensions when dispersed in an aqueous environment [27]. Dispersions of LPN comprise disc-shaped nanoparticles of $1 \mathrm{~nm}$ in thickness and $25 \mathrm{~nm}$ in diameter displaying a positive rim charge and negative surfaces. Clay-based gels have been shown to behave as a functional vehicle for drug retention and delivery with preferential clay-protein interactions [27] and, critically, combinations of LPN with a wide library of polymers [28] offer new bioinks with enhanced printability due to the shearthinning and/or rheology-modifying capability offered by LPN.

We have previously shown that LPN inclusion within an alginate-methylcellulose bioink network improved printability, mechanical stability, limited swelling post crosslinking and preserved cell viability and density [1]. The addition of nanoclay not only enhanced mechanical and physical properties of the resultant bioink, but also modulated the bioink functionality by protein entrapment as a consequence of clayprotein interaction. Gibbs et al. [29] reported on the biofunctionality of LPN gel in association with physiological doses of bone morphogenetic protein-2 (BMP-2) entrapped within the gel. Harnessing the LPN absorptive capacity, BMP-2 was successfully entrapped and remained bioactive, evidenced by ectopic bone formation when the LPN-BMP-2 gels were implanted in vivo. The functional impact of nanoclay dispersion on HMBSCS has recently been detailed [30], with the identification of over 4,000 genes and major cellular pathways (e.g. mitogen-activated protein kinase (MAPK)) modulated by cell-clay interaction. Although LPN inclusion in GelMA hydrogels has been previously reported [31-33], the investigation of its physical properties, printability, functional and clinical potential as a bioink material able to localise angiogenic factors remain unclear. Importantly, high photoinitiator concentrations, post- 
processing with long UV exposure and the application of elevated temperatures present limitations to effective stem cell encapsulation and 3D bioprinting.

Moreover, the current study specifically set out to improve the biofunctionality and biofabrication properties of GeIMA, creating a new multi-functional (osteogenic and angiogenic) nanocomposite bioink for skeletal regeneration therapies. The resulting nanoclay composite bioink could be crosslinked using visible-light and employed to encapsulate HBMSCs, preserving cell viability, proliferation, functionality and the localisation of compounds that are able to promote drug-aided vasculogenesis ex vivo.

\section{Materials and Methods}

\subsection{Gelatin-Methacryloyl synthesis}

Gelatine type A (porcine skin; 300g bloom strength, Sigma-Aldrich) was dissolved in PBS at 10 wt $\%$ concentration. Methacrylic anhydride $(0.6 \mathrm{~g} / \mathrm{ml})$ was added to the gelatine solution and the reaction developed for $1 \mathrm{~h}$ at $50^{\circ} \mathrm{C}$ under constant stirring. The solution was centrifuged and dialysed against deionised water to remove the unreacted methacrylic anhydride. The GelMA solution $\mathrm{pH}$ was adjusted to 7.4 , followed by sterile filtration (0.22 $\mu \mathrm{m}$ filter $)$ and lyophilisation. The degree of modification for methacryloyl substitution was quantified to be $60 \%$ using $1 \mathrm{H}$-proton nuclear magnetic resonance spectroscopy (Bruker Advance $400 \mathrm{MHz}$ ).

\subsection{Nanocomposite synthesis}

Laponite ${ }^{\circledR}$ XLG (LPN, BYK Additives \& Instruments, UK) was chosen as a nanoclay filler to be blended with GelMA. A nanocomposite hydrogel was synthesized using deionised water (DW) (18.2 M $\Omega-\mathrm{cm})$. DW was filter sterilised through a $0.2 \mu \mathrm{m}$ filter (Filtropur S 0.2, Sarstedt) and further exposed to UV for $30 \mathrm{~min}$. Under sterile conditions, 
autoclaved sterile LPN powder was weighted and allowed to disperse in DW with stirring at 300 RPM for 3 hours. Sterile GelMA was added to the LPN suspension and allowed to disperse overnight incubated at $37^{\circ} \mathrm{C}$. The formulations tested correspond to blends of GelMA (5, 7.5 and $10 w t \%)$ and LPN $(0.5,0.75,1,2$ wt\%).

\subsection{Fabrication of LPN-GelMA hydrogel discs}

Three-dimensional discs were fabricated as previously described [12]. Briefly, photoinitiators were added to the different LPN-GelMA formulations and $30 \mu \mathrm{l}$ of the mixtures were transferred to silicone molds ( $\varnothing 5 \mathrm{~mm} \times 1 \mathrm{~mm}$ ), covered with a glass slide and irradiated with visible light (400-450 nm, OmniCure S1500, Excelitas Technologies). Hydrogels were cross-linked using visible light with $1 \mathrm{mM}$ (tris(2,2- bipyridyl) dichlororuthenium (II) hexahydrate (Ru) (Sigma-Aldrich) and $10 \mathrm{mM}$ sodium persulfate (SPS) (Sigma-Aldrich) photoinitiator concentration, irradiating the samples for $3 \mathrm{~min}$ at $3 \mathrm{~mW} \mathrm{~cm}^{-2}$.

\subsection{Physico-chemical and mechanical characterisation}

Mass loss and swelling studies were performed on the fabricated discs to investigate the effect of nanoclay incorporation/into GelMA hydrogels, evaluating hydrogel crosslinking kinetics. Samples were weighed immediately after cross-linking to obtain initial wet mass $\left(m_{\text {initial }}, t 0\right)$, and three samples were lyophilized to obtain their dry weights ( $m_{\text {dry, }}$ $\mathrm{t}=0$ ). The actual macromer fraction was subsequently calculated using equation 1

$$
\text { actual macromer fraction }=\frac{m_{\text {dry }, t 0}}{m_{\text {initial }, t 0}}
$$

The remaining samples were incubated in PBS at $37^{\circ} \mathrm{C}$ to allow swelling and the soluble fraction to leach out of the hydrogel network. After 1 day, the samples were weighed again $\left(m_{\text {swollen}}\right)$. The samples were subsequently lyophilized and weighed a final time 
$\left(m_{\text {dry }}\right)$. The sol fraction was defined as the mass loss after 1 day and was calculated using equation 2 and 3. Mass swelling ratio (q) was calculated using equation 4.

$$
\begin{gathered}
m_{\text {initial,dry }}=m_{\text {initial }} \text { (actual macromer fraction) } \\
\text { sol fraction }=\frac{m_{\text {initial.dry }}-m_{\text {dry }}}{m_{\text {initial,dry }}} 100 \% \\
q=\frac{m_{\text {swollen }}}{m_{\text {dry }}}
\end{gathered}
$$

Compressive Young's modulus was calculated from the linear region of the resulting stress-strain curves (10-15\% strain) obtained with an MTS Criterion ${ }^{\circledR} 42$ mechanical testing machine using a $5 \mathrm{~N}$ load cell. Measurements were performed at room temperature and dry conditions using a pre-load of $0.01 \mathrm{~N}$.

\subsection{Rheology}

Rheological measurements were performed to assess the potential printability of the different LPN-GelMA compositions using a Rheometer (Anton Paar MCR 301) equipped with a plate-plate geometry (gap: $0.3 \mathrm{~mm}$ ). Rotating measurements were conducted using a solvent trap to prevent evaporation. Temperature sweeps from 30 to $1^{\circ} \mathrm{C}\left(1^{\circ} \mathrm{C}\right.$ $\min ^{-1}$ ) were performed at a shear rate of $8 \mathrm{~s}^{-1}$. Viscosity as a function of shear rate was recorded at $20.5^{\circ} \mathrm{C}$ ranging from 0.1 to $1000 \mathrm{~s}^{-1}$. Oscillatory rheological measurements to determine G' and G" moduli were performed at a fixed strain of $1 \%$ and frequency of $1 \mathrm{~Hz}$, across a temperature range from $4^{\circ} \mathrm{C}$ to $40^{\circ} \mathrm{C}$.

\subsection{Fabrication and characterisation of 3D bioprinted LPN- GelMA constructs}

Extrusion-based 3D biopinting of constructs consisting of either GelMA or LPNGelMA was performed using a BioScaffolder (SYS+ENG, Germany). The bioinks were extruded using a computer-aided syringe dispenser with a needle $(\varnothing 300 \mu \mathrm{m})$ at a 
temperature of $20.5^{\circ} \mathrm{C}, \mathrm{XY}$-plane speed of $550 \mathrm{~mm} / \mathrm{min}$, Z-speed of $800 \mathrm{~mm} / \mathrm{min}$, auger speed of $3.5 \mathrm{RPM}$, and a fibre spacing of $1 \mathrm{~mm}$ in a repeating $0-90^{\circ}$ pattern. Scaffolds of 4-10 layers were 3D plotted and irradiated for $5 \mathrm{~min}$ with visible light using the same Ru/SPS photoinitiator concentrations as described above. The constructs were imaged using a 10X air objective on an Axio Imager Z1 microscope (ZEISS) and the diameter of fibres were measured using ImageJ to characterise maintenance of shape fidelity post fabrication and following equilibrium swelling (24h). The change in fibre diameter before $\left(d_{0}\right)$ and after equilibrium swelling $\left(d_{s}\right)$ is given by the equation 5 :

$$
\text { percentage change of fibre diameter }=\frac{d_{0}-d_{s}}{d_{0}} 100 \%
$$

To further characterise printing fidelity, porosity and interconnectivity, the scaffolds were freeze-dried and subsequently sputter coated with carbon (Electron Microscopy Sciences, EMS150T) and imaged via scanning electron microscopy (SEM) using a Jeol JSM 7000F FE-SEM with a secondary electron detection used at an acceleration voltage of $15 \mathrm{kV}$. LPN incorporation in LPN-GelMA was confirmed using energy-dispersive Xray spectroscopy (EDX).

\subsection{BSA and lysozyme absorption and release}

Bovine serum albumin (BSA, Sigma-Aldrich) and Lysozyme from chicken egg (Lysozyme, Sigma-Aldrich) were solubilised in Hank's Balanced salt solution (HBSS, Thermo-Fisher) at $100 \mu \mathrm{g} \mathrm{ml}^{-1}$ and $10 \mathrm{\mu g} \mathrm{ml}^{-1}$ respectively. To correlate the effect of nanoclay inclusion to the absorption capability, nanocomposite (LPN-GelMA) and individual components (LPN and GeIMA) were studied. Previous to absorption, 3D moulds were crosslinked by exposure to $400-450 \mathrm{~nm}$ visible light. Scaffolds $(n=3)$ were soaked in BSA and lysozyme solution for 1, 2, 4, 8, and $24 \mathrm{~h}$. Supernatant was collect to analyse absorption. BSA and lysozyme solutions were replaced with collagenase D (from Clostridium histolyticum, Roche Diagnostics $\mathrm{GmbH}$ ) at $24 \mathrm{~h}$ to simulate in vivo 
conditions and absorption reading carried out at 1, 2, 4, 8 and $24 \mathrm{~h}$ after collagenase addition. BSA and lysozyme were quantified using GloMax Discover microplate reader (Promega).

\subsection{Cell culture, viability and proliferation}

HBMSCs were isolated from patients undergoing routine total hip-replacement surgery with full national ethical approval following patient consent (Southampton General Hospital, University of Southampton, under approval of the Southampton and South West Hampshire Research Ethics Committee (Ref No. 194/99/1)). Unselected HBMSCs were isolated from bone marrow aspirate following as previously described [12]. In brief, bone marrow aspirate was resuspended and washed in alpha modified eagle's medium ( $\alpha-M E M)$ to remove excessive fat. The resulting cell suspension was filtered through a 40- $\mu \mathrm{m}$ cell strainer and layered on LymphoPrep ${ }^{\mathrm{TM}}$ (Lonza) for red blood cell removal via density centrifugation at 2200 RPM (800 G) for 40 min at $18^{\circ} \mathrm{C}$. The bone marrow mononuclear cell portion was then collected at the layered phase between the LymphoPrep ${ }^{\mathrm{TM}}$ and the cell culture medium. The cell suspension was then plated in $175 \mathrm{~cm}^{2}$ cell culture flasks (angled neck, non-pyrogenic polystyrene, Corning, UK) and cultured for cell expansion with a-MEM supplemented with $10 \% \mathrm{v} / \mathrm{v}$ fetal calf serum (FCS), $100 \mathrm{U} \mathrm{ml}^{-1}$ penicillin and $100 \mathrm{~g} \mathrm{ml}^{-1}$ streptomycin. Cell monolayers were maintained at $37^{\circ} \mathrm{C}$ and $5 \% \mathrm{CO}_{2}$ balanced air and passaged at approximately approximatively $80 \%$ cell confluency. Cells were harvested for hydrogel inclusion and experimental protocols using collagenase IV $\left(200 \mathrm{mg} \mathrm{ml}^{-1}\right)$ in serum-free media followed by Trypsin-ethylenediaminetetra- acetic acid (TE) solution treatment. 


\subsection{Functionality assays on HBMSC-laden 3D bioprinted constructs}

HBMSCs (P3) were incorporated into sterile LPN-GeIMA and photoinitiator solutions at a concentration of $5 \times 10^{6}$ cells $\mathrm{ml}^{-1}$ and used as a bioink for scaffold 3D fabrication as described above. HBMSC-laden scaffolds were cultured in either osteogenic differentiation media, consisting of a-MEM supplemented with $10 \%$ FBS, $1 \%$ Penicilin/Streptomycin, 20mM Ascorbic Acid, 10mM $\beta$-Glycerophosphate/(SigmaAldrich) and 10nM Dexamethasone (Sigma-Aldrich) or in the same composition media excluding the osteogenic inducing factor Dexamethasone. After 21 days of culture, the printed scaffolds were washed with PBS, fixed with 10\% neutral buffered formalin for 1 hour and washed with deionized water. To visualize mineralized nodule formation, the scaffolds were incubated in alizarin red $\mathrm{S}$ solution $(\mathrm{pH} 4.2$, Sigma-Aldrich) for 15 seconds. The staining solution was removed and the cells were washed several times with deionized water to remove the non-specific excess stain. Images were captured using a 10x air objective on an Axio Imager Z1 mícroscope (ZEISS).

\subsection{Chorioallantoic membrane (CAM) assay}

Animal procedures were undertaken in accordance with the guidelines and regulations of the Animals Act 1986, UK. The chorioallantoic membrane protocols were conducted under Home Office Approval UK (Project licence - PPL 30/2762). Chicken eggs were incubated in a Hatchmaster incubator (Brinsea, UK) for 10 days at $37^{\circ} \mathrm{C}$ in a $60 \%$ humidified atmosphere and 1 hour rotation. After 10 days post-fertilisation, a scalpel was used under sterile conditions to create a $2 \mathrm{~cm}^{2}$ window on the eggshell. VEGF was absorbed at $10 \mu \mathrm{g} \mathrm{ml}^{-1}$ onto 3D casted discs. Three-dimensional scaffolds $(n=10)$ were inserted in chick egg and evenly distributed onto chorioallantoic membrane. Eggs were sealed with sterile Parafilm and incubated without rotation. Candling was undertaken daily to inspect chick embryos. After 7 days of incubation, 3D samples were harvested and CAM integration inspected with a stereomicroscope with digital camera 
(Canon Powershot G2). Fully developed chick embryos were recorded and gestational process terminated under specific Home Office guidelines.

\subsection{Histological analysis}

Samples collected from the CAM assays, were embedded in optimal cutting temperature (OCT) compound and stored at $-80^{\circ} \mathrm{C}$. Cryostat (CM 1850, Leica) was used to produce tissue sections of $8 \mu \mathrm{m}$. Kawamoto film's method [34] was used to collect slices and samples were stained on the tape. Goldner's Trichrome (GT) involved the use of Weigert's haematoxylin, ponceau-fuchsin-azophloxin (erythrocytes), phosphomolybdic acid (cytoplasm), and light green (collagen). Sections were then mounted with super cryomounting medium type R3 (SCMM R3, Section lab Co.Ltd, Japan) before imagining with Zeiss Axiovert 200.

\subsection{Statistical analysis}

Experimental studies were evaluated by one-way and two-way ANOVA using Bonferroni's multiple comparison tests. Analysis was carried out with GraphPad Prism 7 and significance set at $p<0.05$. 


\section{Results}

\subsection{Physico-chemical and mechanical properties of clay-based} nanocomposite hydrogels
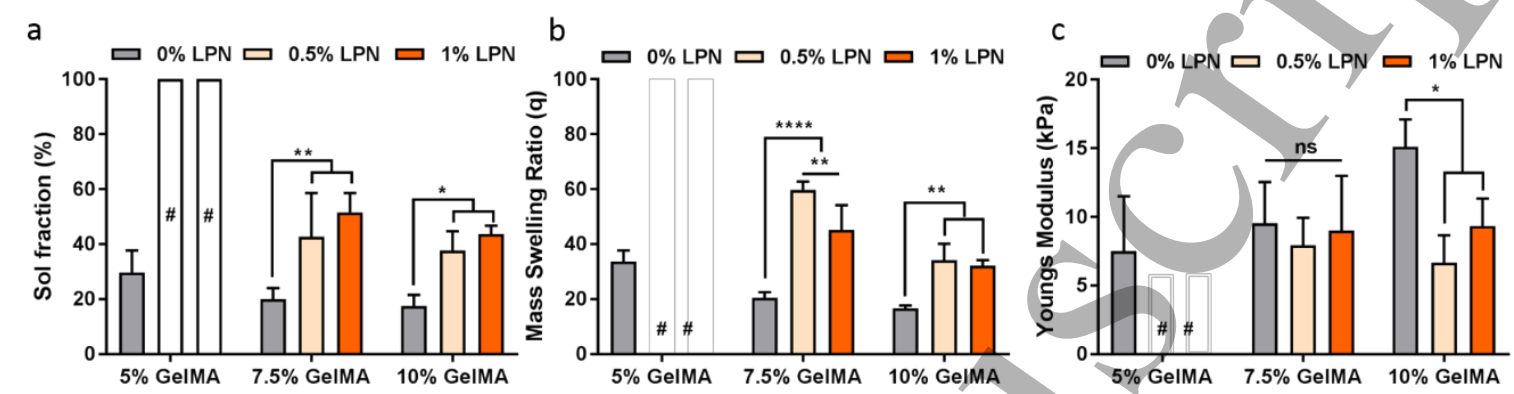

Figure 1. Physicochemical characterisation of LPN-GelMA hydrogels. The distinct hydrogel formulations were evaluated for (a) soluble fraction, (b) mass swelling ratio and (c) mechanical strength. Mean $\pm S D, n=3 * \star \star \star ~(p<0.0001) ;{ }^{\star *}(p<0.01) ;{ }^{\star}(p<0.05)$. \# indicates absence of hydrogel after $24 \mathrm{~h}$ incubation.

The effect of LPN on the crosslinking process of GelMA was studied using mass loss and swelling experiments. The efficiency of hydrogel photo-polymerisation reactions can be affected by environmental conditions or by the presence of organic and inorganic components. The calculated soluble fraction indicates the crosslinking efficiency of the system by measuring the amount of non-crosslinked components. Incorporation of LPN interfered with the crosslinking of the GeIMA macromer as revealed by an increase $(p<0.01, p<0.05)$ in soluble fraction in all nanocomposite formulations examined (Figure 1a). This was evident for 5 wt\% GelMA, with the absence of gel formation (or formation with rapid disintegration) upon addition of 0.5 and 1 wt $\%$ LPN. For 7.5 and 10 wt\% GelMA, there was a significant increase $(p<0.01, p<0.05)$ in sol fraction resulting from the addition of 0.5 and 1 wt $\%$ LPN. The soluble fraction increased irrespective of the final LPN concentration, as well as an increase in the mass swelling ratio of the nanocomposite hydrogels as a consequence of the formation of a loose hydrogel network. Inclusion of 1 wt $\%$ LPN in 7.5 wt $\%$ GelMA led to a significantly $(p<0.01)$ reduced 
swelling ratio in comparison to inclusion of $0.5 \mathrm{wt} \%$ LPN (Figure 1b). The compressive Young's modulus of the LPN-GelMA nanocomposite at 7.5 and $10 \mathrm{wt} \%$ showed comparable mechanical stiffness, irrespective of the LPN concentration employed. While 10 wt $\%$ GelMA showed a lower moduli $(p<0.05)$ upon 0.5 and 1 wt $\%$ LPN addition, 7.5 wt\% GelMA preserved its mechanical properties regardless of LPN addition. (Figure 1c).

\subsection{Nanocomposite hydrogels show higher drug retention and localisation within the 3D matrix}

a

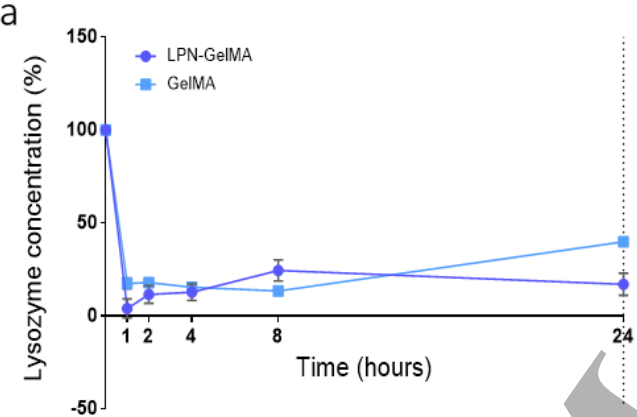

c

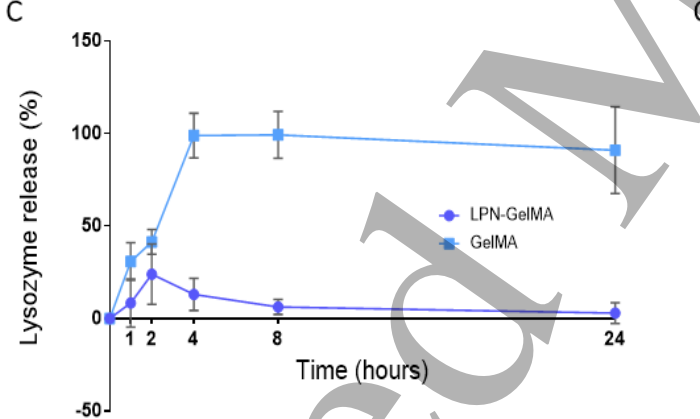

b
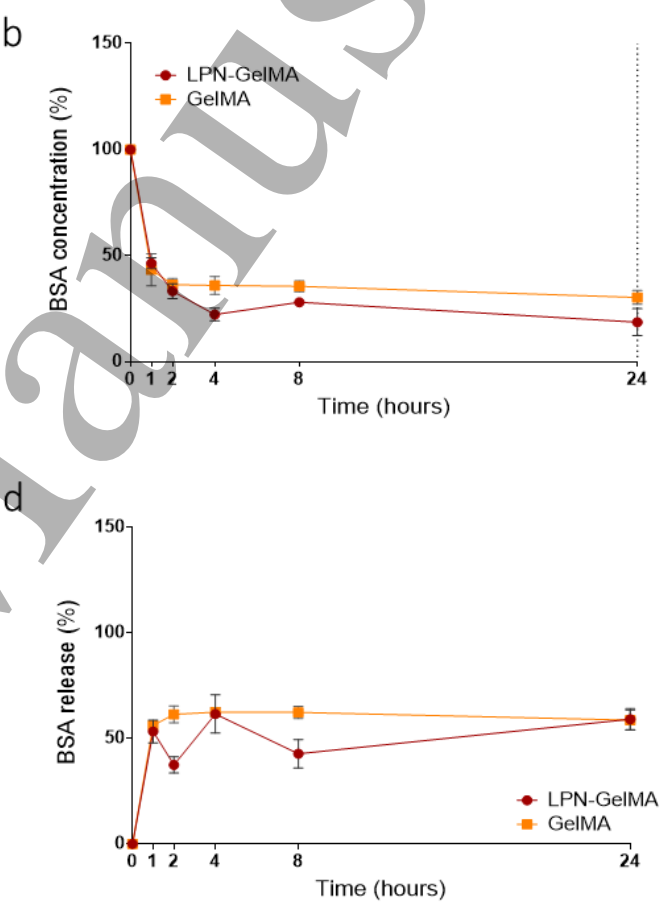

Figure 2. Concentration of absorbed lysozyme and bovine serum albumin (BSA) and subsequent induced release from nanoclay composite scaffolds. Collagenase solution addition indicated by dotted line at $24 \mathrm{~h}$ after initial incubation. Lysozyme (a) and BSA (b) absorption on LPN-GeIMA and GelMA scaffolds. Collagenase-mediated release of lysozyme (c) and BSA (d) from LPNGeIMA and GeIMA. The percentage concentration refers to the percentage of drug absorbed $(a, b)$ and then percentage of drug release $(c, d)$.

The potential of LPN-GelMA hydrogels to localise compounds of interest was evaluated by absorption and immobilisation of drug analogues into casted hydrogel discs (Figure S1 a,b). The absorption of lysozyme into LPN-GelMA and GelMA discs resulted from the reading after 1, 2, 4, 8 and 24 hour of the protein suspension in which discs were absorbed. 
A rapid decrease of lysozyme concentration in the supernatant up to $3.9 \pm 5.1 \%$ and $11.4 \pm 4.7 \%$ of the total initial protein concentration in suspension $\left(10 \mu \mathrm{g} \mathrm{ml}^{-1}\right)$ was observed after only 1 hour for LPN-GelMA and GelMa respectively (Figure 2a). The incorporation of $1 \mathrm{wt} \%$ LPN did not significantly affect the lysozyme absorption capability, and both LPN-GelMA and GelMA showed a rapid absorption of the lysozyme from the solution reaching a rapid saturation state. In contrast, the presence of $1 \mathrm{wt} \%$ LPN in GelMA gels significantly increased BSA absorption capacity compared to nanoclay-free GelMA gels. The concentration of BSA (initial loading of $100 \mu \mathrm{g} \mathrm{ml}^{-1}$ ) in the supernatant of LPN-GelMA gels $(18.63 \pm 6.4 \%)$ was found to be significantly lower than GelMA $(30.3 \pm 3.3 \%)$ upon saturation after 24 hours, indicating that greater amount of BSA was absorbed into the LPN-GelMA samples (Figure $2 b$ ).

The ability of LPN-GeIMA and GelMA gels to retain the absorbed lysozyme or BSA was determined. Collagenase solution $\left(1 \mathrm{mg} \mathrm{ml}^{-1}\right)$ was used to simulate in vivo digestion conditions, where total release of the absorbed lysozyme was observed in the GelMA samples after 4 hours in the digestion bath, reaching a plateau with continuous release up to 24 hours $(90.9 \pm 23.5 \%$ ) (Figure 2 c). Only a negligible amount of lysozyme was released from LPN-GelMA gels (3.1 $\pm 5.2 \%)$ even after 24 hours, demonstrating effective drug localisation within the gel as a consequence of the presence of LPN. Similarly for BSA, collagenase digestion induced the rapid release of BSA from GeIMA discs after only 2 hours (Figure $2 d$ ), plateauing at $58.6 \pm 4.7 \%$ after 24 hours. In contrast, while LPN-GelMA gels displayed an immediate release of BSA after 1 hour, a subsequent reduction in released concentration was measured after 2 hours, with a final concentration of $59.1 \pm 5.1 \%$ after 24 hours, illustrating the significant retention capacity of LPN-GeIMA. 


\subsection{Printability of nanocomposite scaffolds is ameliorated by} nanoclay addition

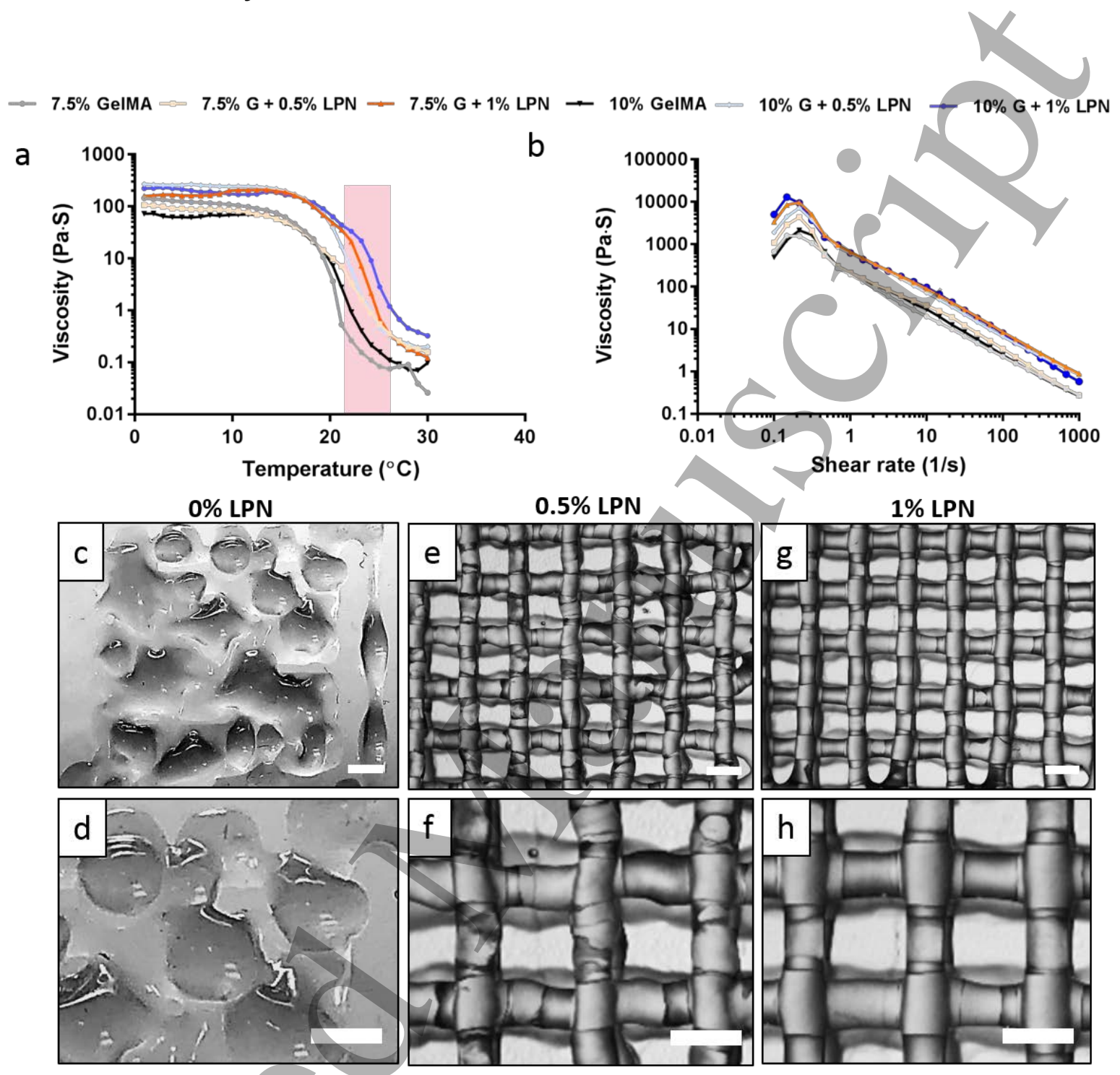

Figure 3. Printability of LPN-GelMA scaffolds. Rheological characterization showing (a) temperature sweep at constant shear rate (8/s) (printing window highlighted in red) and (b) viscosity as a function of shear rate for various LPN-GelMA formulations at $20.5^{\circ} \mathrm{C}$. Comparison of printing fidelity achieved by $(\mathrm{c}, \mathrm{d}) 7.5 \mathrm{wt} \%$ GelMA and $7.5 \mathrm{wt} \%$ nanocomposite bioinks incorporating (e,f) $0.5 \%$ LPN and $(g, h) 1 \mathrm{wt} \%$ LPN. (Scale bars: $1 \mathrm{~mm}$ ).

Temperature sweeps were performed to evaluate changes in bioink viscosity at a given fixed shear rate comparable to that experienced during the bioprinting process. The printability window for the different formulations, determined as the temperature range in which the physical sol-gel transition occurs, was observed to reside between 20 and $25^{\circ} \mathrm{C}$. Incorporation of LPN notably increased the viscosity of GelMA in a concentration dependent manner. Formulations incorporating $1 \mathrm{wt} \% \mathrm{LPN}$ displayed the 
highest viscosity, with the lowest viscosity recorded for nanoclay-free samples (Figure 3a). Incorporation of LPN did not affect the shear-thinning property of the bioinks and all evaluated compositions demonstrated a gradual decrease in viscosity with increasing shear rate. Strikingly, at any given shear rate, compositions with higher LPN concentration displayed an associated higher viscosity (Figure 3b). Furthermore, storage (G") and loss (G') moduli of GelMA solutions increased upon addition of $0.5 \%$ and $1 \%$ LPN. (Figure S2).

Bioprinted scaffolds, without LPN addition, showed poor filament formation and print fidelity, with an absence of filament stacking (Figure 3c,d). In contrast, incorporation of LPN (0.5 and 1 wt\%) significantly improved printability of the bioinks resulting in highquality $3 \mathrm{D}$ printed scaffolds, evidenced by visibly improved filament formation and stacking with excellent maintenance of shape fidelity following photocrosslinking (Figure $3 e-h)$. It should be noted that addition of elevated LPN concentrations ( $>2$ wt $\%$ ) resulted in a highly viscous blend that did not allow adequate photoinitiator incorporation and an inability to form laminar filaments upon extrusion. A summary of the formulations tested in relation to their printability can be find in Figure S3. 


\subsection{Printed nanocomposite constructs exhibit interconnectivity} and porosity retention with nanoclay inclusion

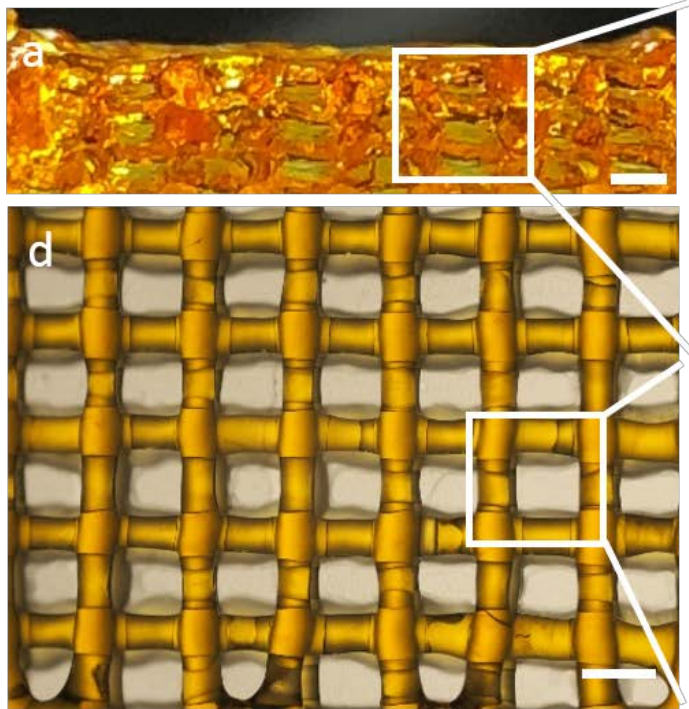

g

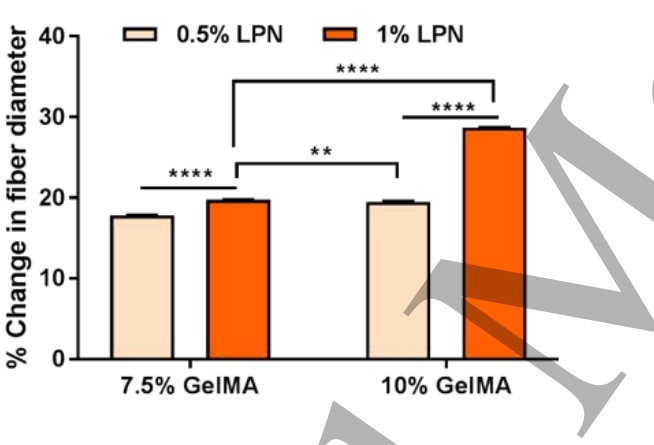

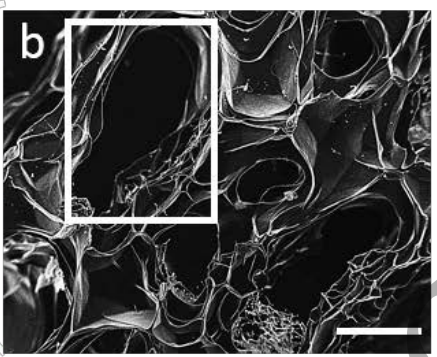
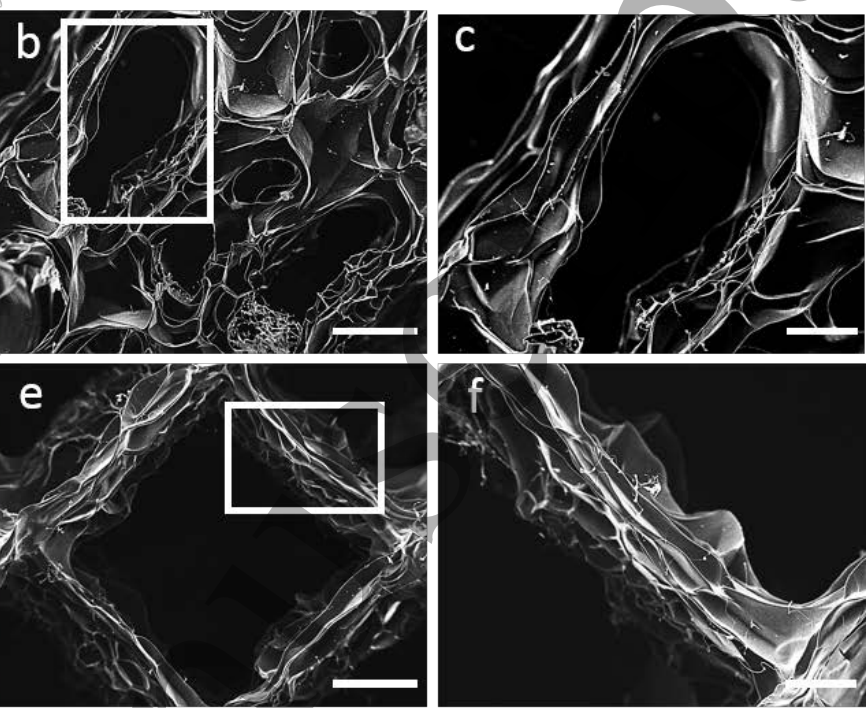

$\mathrm{h}$

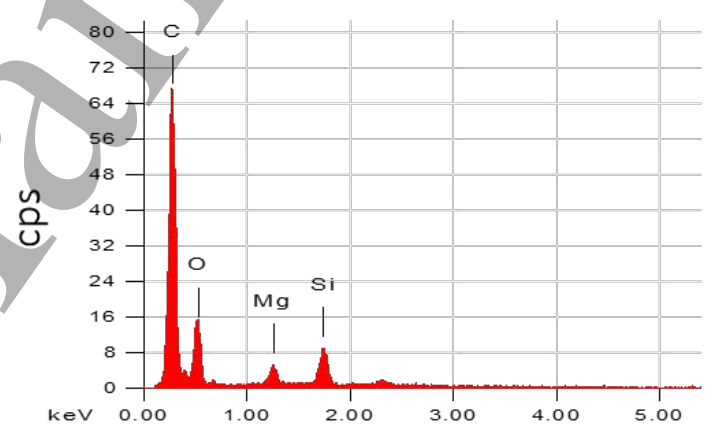

Figure 4. Characterisation of $1 \%$ LPN - 7.5\% GelMA 3D printed scaffold through (a,d) optical imaging; (b,c,e,f) scanning electron microscopy, fibre diameter measurements after equilibrium swelling (g) compared to 0.5\% LPN inclusion and (h) energy dispersive X-ray spectroscopy.

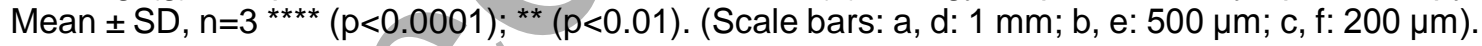

The 3D printed nanocomposite constructs showed adequate porosity retention in the $z$ (Figure 4a-c) and $x-y$ (Figure 4d-f) axis resulting in an interconnected pore network. SEM micrographs (Figure 4c,f) confirmed an open pore morphology throughout the 3D printed LPN-GelMA strands. EDX analysis (Figure 4h) confirmed the incorporation of LPN, evidenced by the presence of distinctive silica and magnesium peaks. Changes in fibre diameter post swelling (Figure $4 \mathrm{~g}$ ) were assessed as the subsequent step in shape fidelity retention, simulating the behaviour of the printed construct in an in vivo environment. The constructs successfully retained their interconnected lattice structure post-swelling as well as filament fidelity, displaying a significant $(p<0.0001)$ change in 
fibre diameter (<20\%) for 7.5 wt\% GelMA incorporating 0.5 and 1 wt $\%$ LPN, as well as 10 wt $\%$ GelMA incorporating 0.5 wt $\%$ LPN. In contrast 10 wt $\%$ GelMA - 1 wt $\%$ LPN showed a greater change in fibre diameter post swelling compared to 7.5 wt $\%$ GelMA $1 \mathrm{wt} \%(p<0.0001)$.

\subsection{Cell-laden nanocomposite constructs preserved cell} viability, supported proliferation and display osteogenic capacity in vitro

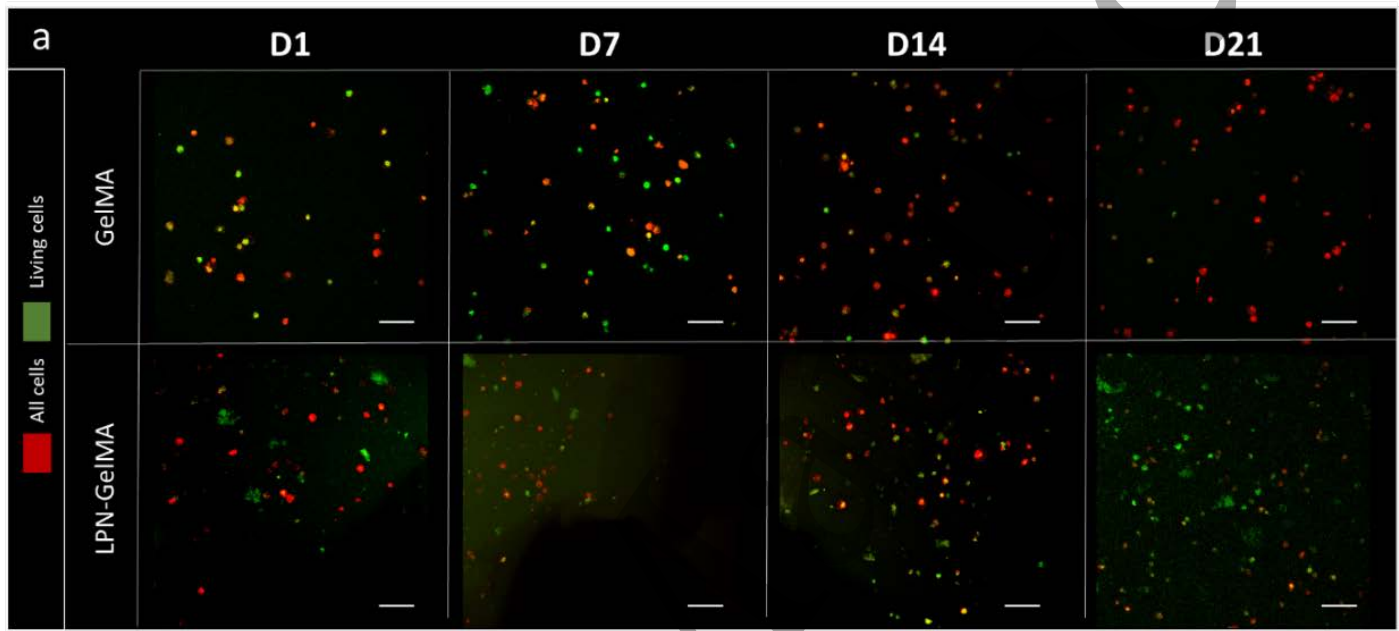

b
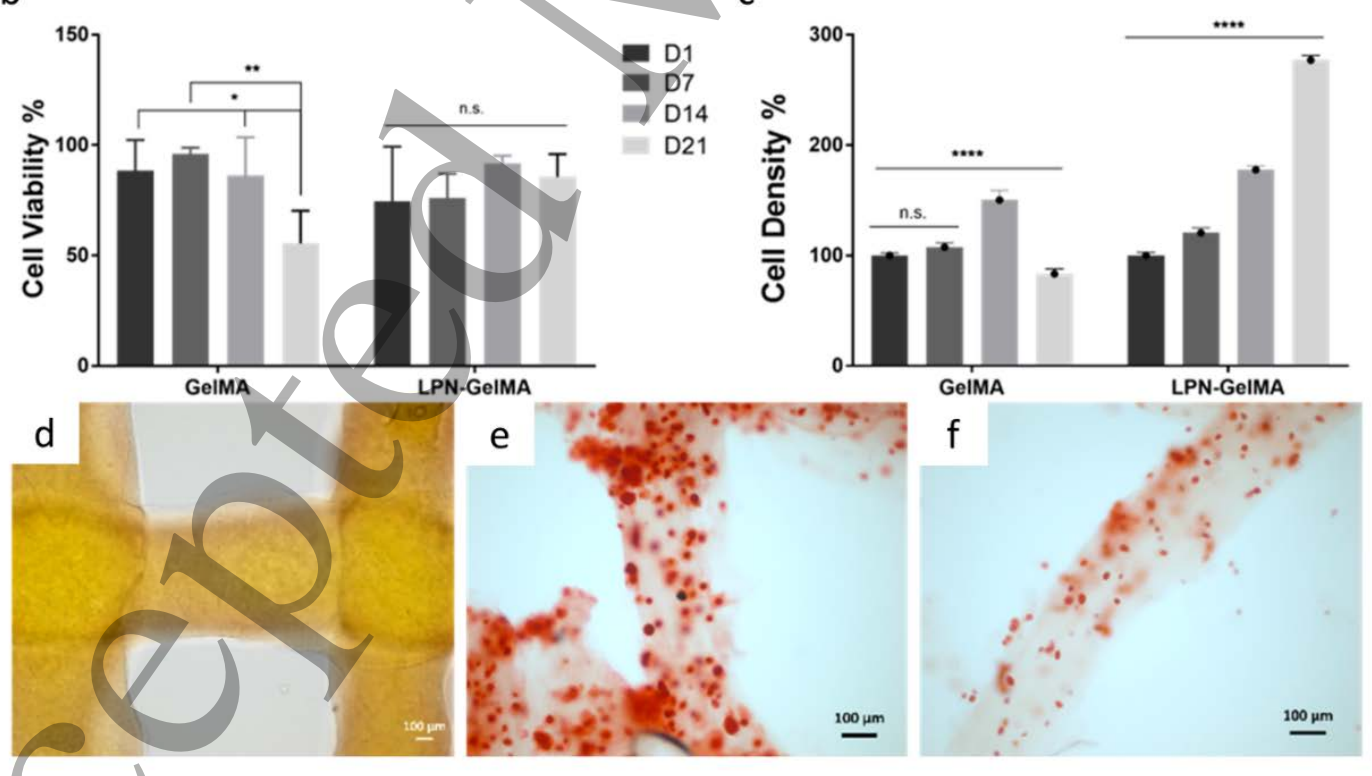

Figure 5. Cell viability, proliferation and osteogenic capacity of HBMSC-laden 3D nanocomposite constructs. (a) Confocal micrographs illustrate maximum intensity projection of HBMSCs-laden 3D LPN-GelMA and GeIMA scaffolds cultured up to 21 days. Encapsulated HBMSCs in GelMA and LPN-GelMA were stained for mitotically active cells at 1, 7, 14 and 21 days. Cells were prelabelled with DiD lipid-labelling dye (red) to stain all cells prior to encapsulation and printing. Living cells were stained with Calcein AM (green). Non-viable (dead) cells, viewed as red within 
the cell population in contrast to viable (green) Calcein-AM stained populations.(Scale bar: 200 $\mu \mathrm{m})$. Cell viability percentages (b) were calculated as the difference between living and all cells. Cell density percentages (c) calculated from living cells normalised to volume of interest. Alizarin Red staining of (d) non-cell laden nanocomposite scaffolds and HBMSCs-laden scaffolds cultured with (e) full osteogenic media and (f) without Dexamethasone. Mean $\pm S D, n=3$ $\star \star \star \star *(p<0.0001),{ }^{* \star}(p<0.01),{ }^{*}(p<0.05)$.

HBMSCs were encapsulated in LPN-GelMA bioink for initial assessment of cell viability, survival and proliferation following extrusion 3D bioprinting (Figure 5a). Cells encapsulated in GelMA displayed high viability after $24 \mathrm{~h}(88.40 \pm 13.89 \%)$, which increased after 7 days $(95.88 \pm 2.90 \%)$, although cell proliferation was observed to significantly decrease $(p<0.05)$ over time after $14(86.26 \pm 17.22 \%)$ and 21 days $(55.54$ $\pm 14.72 \%$ ) (Figure 5b). In contrast, HBMSCs encapsulated within LPN-GelMA exhibited high cell viability after $24 \mathrm{~h}(74.44 \pm 24.89 \%)$ and remained stable with negligible change in cell viability observed over 7 days $(76.12 \pm 10.93 \%)$ and up to 21 days of culture. Cell density results (Figure 5c) showed elevated initial proliferation of HBMSCs encapsulated in GelMA controls at 7 days (107.57 $\pm 3.86 \%)$ and up to 14 days (150.36 $\pm 8.58 \%)$ of culture, but significantly decreased after 21 days of culture (83.36 \pm 4.57 \%). In contrast, HBMSC-laden LPN-GelMA scaffolds presented a sustained increase in cell density over $7(120.55 \pm 4.46 \%), 14(177.74 \pm 3.30 \%)$ and up to 21 days of culture $(276.91 \pm 4.28 \%)$. The increase in density of cells encapsulated in LPN-GelMA is evidence of HBMSCs proliferation over time.

To determine the ability of the nanocomposite bioinks to support osteogenic differentiation, HBMSCs-laden constructs were bioprinted and cultured for 21 days in osteogenic media (with dexamethasone) or in basal media (absence of dexamethasone). Non cell-laden scaffolds did not retain the Alizarin Red staining, showing no uptake from the LPN alone (Figure $5 \mathrm{~d}$ ). LPN-GeIMA bioinks showed the capacity to support osteogenic differentiation of HBMSCs, evidenced by extensive mineral deposition (Figure 5e). Interestingly, cell-laden constructs cultured in the absence of dexamethasone (Figure 5f) also displayed areas of mineralisation, 
suggesting an osteogenic response from the nanoclay throughout the bioprinted fibres, although at an apparent lower rate compared to dexamethasone supplementation.

\subsection{Implantation of LPN-GeIMA in chick chorioallantoic} membrane model results in vascularisation and sample integration
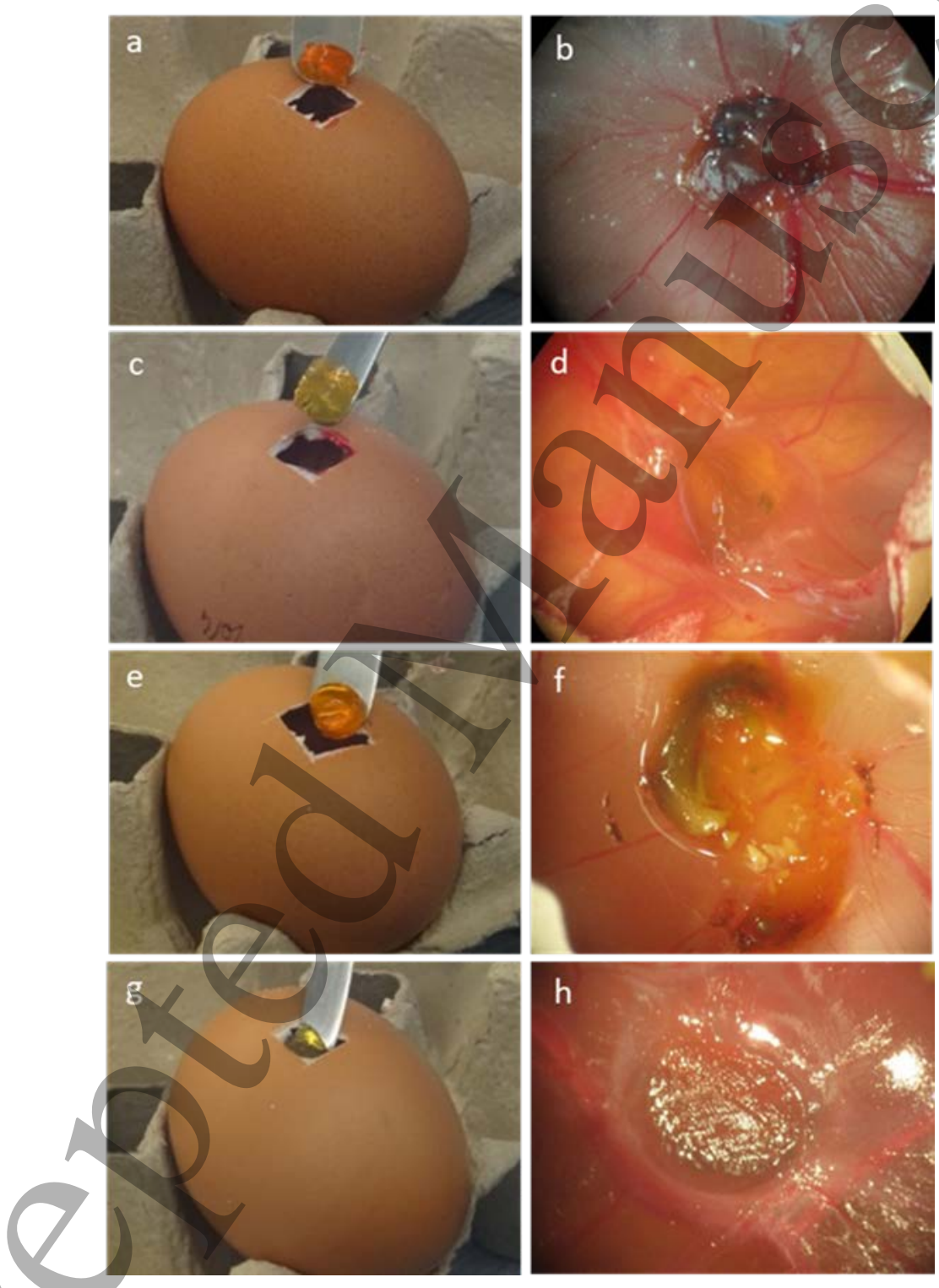

Figure 6. Chicken chorioallantoic membrane ex vivo model implantation of LPN-GelMA and GelMA 3D hydrogels. Macrographs of implantation of VEGF-LPN-GelMA (a), VEGF-GelMA (c), LPN-GelMA (e), and GelMA (g) confirmed consistency of the implantation. In situ macrographs of VEGF-LPN-GeIMA (b), VEGF-GeIMA (d), LPN-GelMA (f) and GeIMA (h) confirmed integration of all the scaffolds, with enhanced vascularisation in VEGF-LPN-GeIMA (b) and VEGF-GelMA (d) as expected. 
The CAM model was used to investigate the ability of LPN-GelMA hydrogel discs (Figure S4) to retain pro-angiogenic factors and to subsequently stimulate vasculogenesis compared to nanoclay-free GelMA.

LPN-GeIMA-VEGF (Figure 6a), GeIMA-VEGF (Figure 6c), LPN-GeIMA (Figure 6e) and GelMA (Figure 6g) hydrogels were implanted in 10-day staged CAM eggs. After 7 days of incubation at $37^{\circ} \mathrm{C}$, LPN-GelMA-VEGF (Figure 6b), GelMA-VEGF (Figure 6d), LPNGelMA (Figure 6f) and GelMA (Figure 6h) samples were observed to be fully integrated into the CAM and highly vascularised.
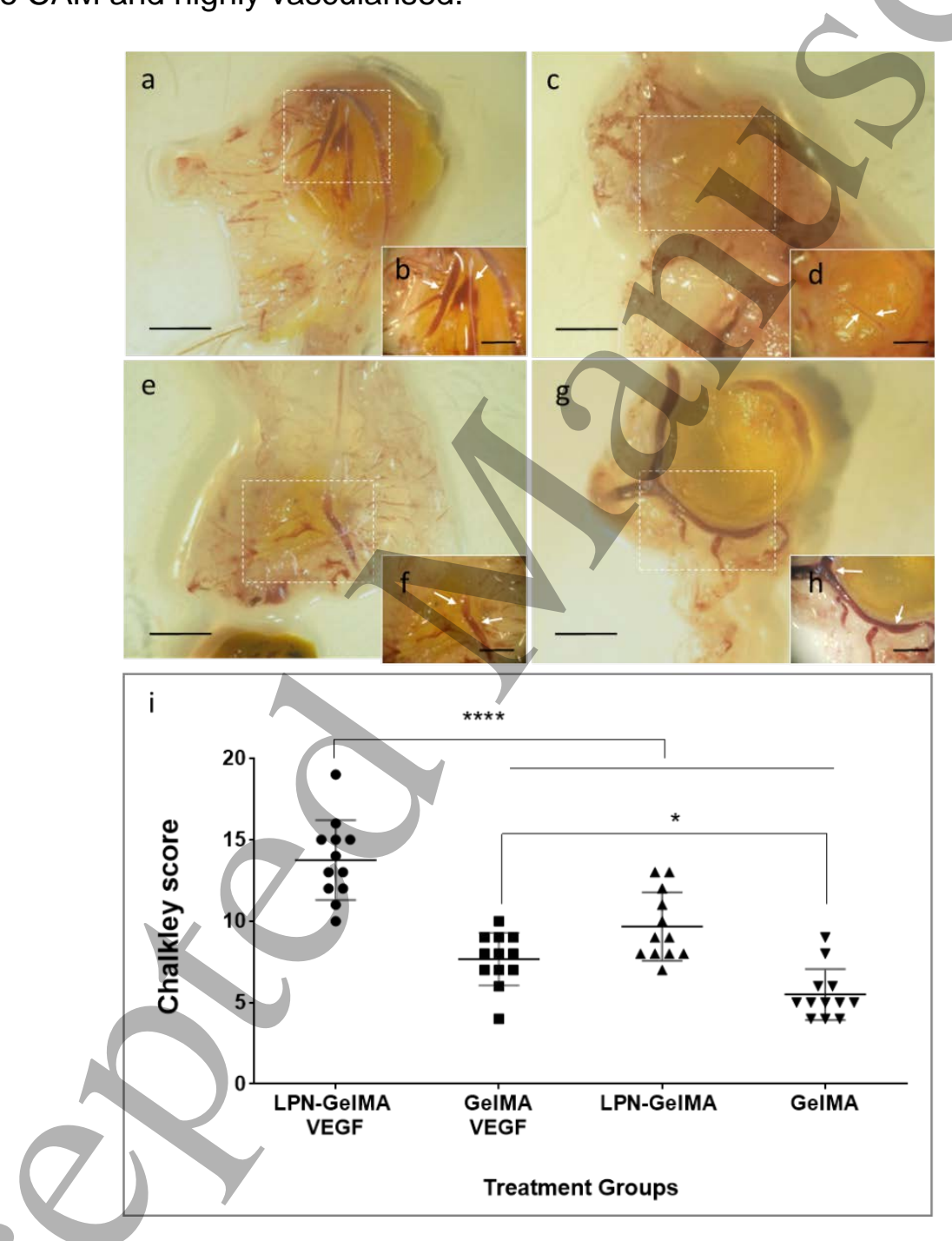

Figure 7. Vascular integration following implantation in CAM. (a) VEGF-absorbed LPN-GelMA 3D scaffolds show integration with the chorioallantoic membrane. (b) Macrograph of intricate vasculature (arrows) surrounding the sample. (c) VEGF-GeIMA scaffolds demonstrating integration and (d) vascularisation (arrows). (e) LPN-GelMA scaffold integrated well with surrounding tissue and (f) presented blood vessel penetration as indicated by arrows. (g) GelMA 3D construct displayed limited penetration by surrounding vasculature but $(\mathrm{h})$ with thick blood vessel circumnavigating the sample (arrows). Chalkley score (i) analysis. Mean $\pm S D, n=12$ ${ }^{* * * *}(p<0.0001),{ }^{*}(p<0.05)$. 
LPN-GeIMA-VEGF (Figure 7a) showed extensive integration evidenced by vasculature (Figure 7b) penetrating through the 3D construct. GeIMA-VEGF (Figure 7c) displayed a reduced degree of vascular penetration (Figure 7d). The LPN-GelMA VEGFfree construct (Figure 7e) was noted to be fully integrated with the CAM, with new vasculature covering the construct (Figure 7f). GelMA (Figure 7g) implants were observed to integrate with the CAM, however major vessels were observed to be wrapped around the periphery and no distinct penetration of vessels into the material observed (Figure 7h). Chalkley score (Figure 7i) confirmed significantly higher vascular penetration in VEGF-absorbed LPN-GeIMA compared to GeIMA-VEGF, LPN-GeIMA and GeIMA $(p<0.0001)$. Addition of VEGF did not show any significant differences when absorbed into GelMA scaffolds compared to VEGF-free LPN-GelMA, although a significant difference $(p<0.05)$ compared to VEGF-free GelMA was noted.

Histological analysis (Figure 8) further confirmed that VEGF absorbed LPN-GelMA (Figure $8 \mathrm{I}$ ) a-h) had a significant effect on CAM integration and vessel penetration within the 3D scaffold compared to VEGF absorbed GeIMA (Figure 8 II) a-h). In particular, LPN-GelMA-VEGFconstructs showed CAM integration around the sample periphery (Figure 8 I) a-f) with vessel adherence and infiltration (Figure 8 I) g,h). GelMA discs absorbed in VEGF and implanted (Figure $8 \mathrm{II})$ a-h) showed CAM integration (Figure 8 II) a-d) with membrane tissue penetration and close proximity of blood vessels (Figure 8 II) e-h) but with no sign of vessel penetration. LPN-GelMA controls (Figure 8 III) a-h) showed major vessels in close proximity (Figure 8 III) a-d) with clear integration with the surrounding CAM and a microporous internal structure that showed blood vessels penetration and integrátion (Figure 8 III) g,h). GelMA controls (Figure 8 IV) a-h) presented signs of CAM integration (Figure $8 \mathrm{IV}$ ) a,b) and blood vessel proximity (Figure 8 IV) c,d). Partial integration with the vascularised membrane was observed but with major separation gaps surrounding GeIMA samples. 
I) LPN-GeIMA + VEGF
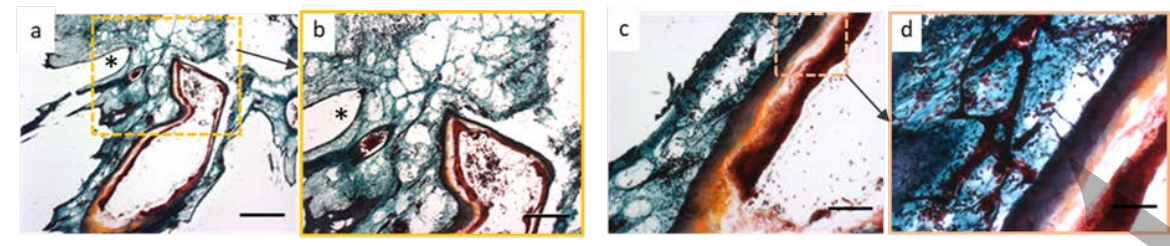

II) GeIMA + VEGF
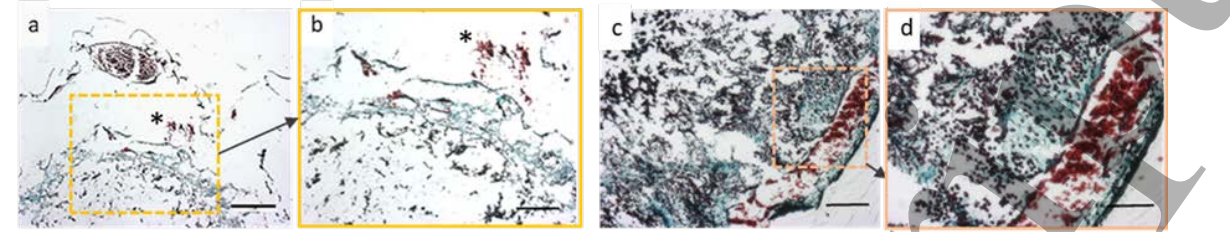

III) LPN-GeIMA
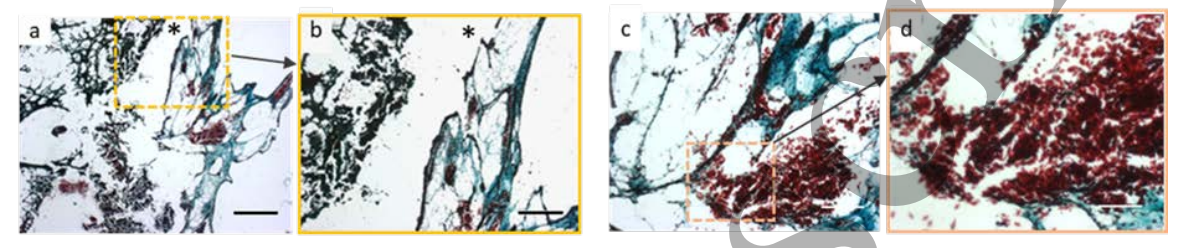

IV) GelMA
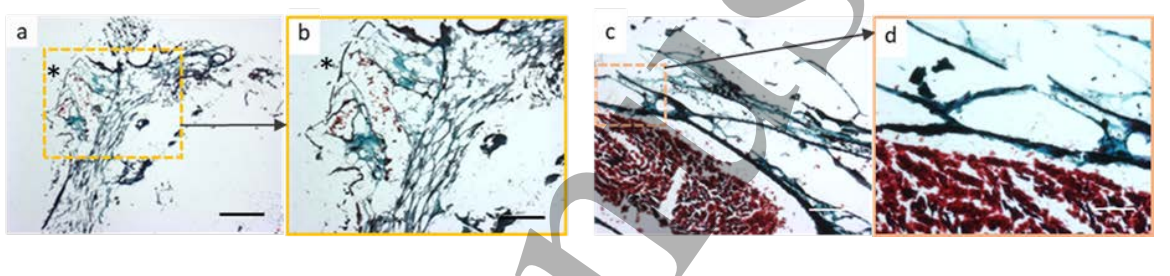

Figure. 8. Histological analysis of VEGF-absorbed or free LPN-GelMA and GelMA in CAM model. Goldner's Thrichrome staining showing erythrocytes in red, collagen in green. H\&E staining showing nuclei in black. I) LPN-GelMA scaffolds with VEGF (a,b) resulted highly integrated with the CAM membrane. Orange photoinitiators entrapped within LPN-GelMA outer surface. (c-f) Major blood vessels in proximity to the integrated scaffold edges (dark black). $(g, h)$ CAM vessels deeply integrated with the scaffold. II) GelMA scaffolds with VEGF $(a, b)$ were found in close proximity of major vessels. (c-f) GelMA scaffold porous structure (black) displayed integration with the CAM membrane. $(g, h)$ Vessels and CAM (light green) integration with GelMA throughout the entire scaffold perimeter. III) LPN-GelMA scaffolds (a-d) integrated with the membrane. (e) Major blood vessels in the vicinity of the LPN-GeIMA (f) scaffolds loaded with orange photoinitiator. Blood vessels were found in the outer proximity $(\mathrm{g})$ and internalised $(\mathrm{h})$ in the scaffolds. IV) GelMA scaffolds (a,b) integrated with small (c) and large vessels (d) closely integrated on the outside of the scaffolds. Close integration resulted evident in close proximity of vessels (e) and small portion of CAM (f). GelMA scaffolds showed a less compact structure and integrated (g,h) compared to VEGF absorbed samples Scale bars: I) II) III) IV) a,b: $500 \mu$ m; I) II) III) IV) c,d: $250 \mu \mathrm{m}$ I) II) III) IV) e,f: $100 \mu \mathrm{m}$ I) II) III) IV) g,h: $50 \mu \mathrm{m}$ 


\section{Discussion}

Biofabrication approaches aim to harness cells, biological factors and 3D bioprinting technology to recapitulate the native structural organisation of tissues, in order to fabricate functional tissues that are suitable for implantation and integration with surrounding host tissue. As such, there is a need to develop bioinks that are able to localise stem/progenitor cells without inducing cell damage, maintain shape fidelity postfabrication and, critically, tailored for tissue specific regeneration. Gelatin-based hydrogels have emerged as popular bioink candidates, given their rheological properties and excellent cytocompatibility [35]. In particular, gelatin methacryloyl (GeIMA), has proved to be a versatile platform given the potential to tailor the physicochemical and mechanical properties of the material [36]. Thus, GelMA presents an attractive functional bioink system with the potential to sustain cell viability [7] and deliver viable cells in a hierarchichal 3D organisation [16]. However, the printability of GeIMA remains limited due to its narrow biofabrication window. Here, GelMA is only tuneable within a narrow temperature-controlled range as a consequence of the thermosensitivity disruption due to gelatin fragmentation during the functionalisation process [37].

Extrusion-based 3D bioprinting of GelMA has been widely attempted $[16,38]$ and in almost all cases, the printability has been shown to be improved by the addition of several additives including collagen [12], PEG [39] and gellan gum $[16,40]$ to create cellladen bioinks applied to cartilage $[16,41]$, bone $[42,43]$ and vascular $[44,45]$ tissue engineering, However, as detailed, none of the additives used in previous studies has targeted the bioprinting of multi-functional cell laden bioinks that combine both skeletalspecific (osteogenic and angiogenic) requirements to stimulate bone tissue formation and blood vessel ingrowth. Bioprinting of cell-laden GelMA bioinks can be facilitated by modulation of temperature and post-printing light curing [46]. However, conventional UV light crosslinking is known to be toxic for encapsulated cells [10], while temperature 
regulation can impact on the control of fibre deposition. A recent approach [12] has reported on the application of a visible-light photoinitiating system to better preserve cell viability while crosslinking a 3D printed structure. Regardless, precise temperature control remains the main parameter in the regulation of GeIMA deposition. To overcome this limitation, the viscoelasticity of GelMA can be influenced by mixing with a nano-filler, further widening the biofabrication window and, critically, removing the need for precise temperature control for extrusion bioprinting.

Previous studies on LPN-GelMA composites have shown the beneficial integration of nanoclay particles within GelMA matrices with enhanced physical, chemical and biological properties [19]. Xavier et al. [31] first described the interaction of nanoclay with GelMA hydrogels, demonstrating cell functionality of mouse pre-osteoblasts on 2D hydrogel surfaces and the shear thinning behaviour of the composite, thus illustrating the potential of LPN-GeIMA as a suitable candidate bioink. Paul et al. [32] showed that incorporation of LPN within GelMA induced the in vitro osteogenic differentiation of encapsulated HBMSCs. In addition, the composite was observed to be biocompatible in vivo upon subcutaneous implantation in an immunocompetent rat model. Here, we sought to characterise the LPN-GeIMA blend by systematically characterising the physical properties, printability and biofabrication window of multiple light-curable LPNGeIMA bioink formulations. The current studies demonstrate the multi-functional capacity of incorporating LPN in GelMA to facilitate bioprinting of scaffolds with high shape fidelity, cell viability and the potential to stimulate osteogenic differentiation, but, importantly, to also localise growth factors and stimulate vascular network penetration ex vivo. Therefore, for the purposes of this study - and in relation to the current definition of bioinks [4] - we suggest that both non cell-laden LPN-GelMA and cell-laden LPNGelMA are considered as bioinks given that both formulations contain biologically active components (e.g. LPN can release or bind growth factors) and/or cells, and both formulations can be extrusion bioprinted. 
Recently, Chimene et al. [33] developed a bioink containing LPN, GeIMA and the polymer kappa-carrageenan ( $\mathrm{kCA}$ ) which allowed bioprinting of tall multi-layered structures that maintained high cell viability (>90\%). The authors reported the addition of LPN and kCA resulted in over 4-fold increase in GelMA hydrogel stiffness (from 16.5 $\pm 1.5 \mathrm{kPa}$ to $71.1 \pm 4.9 \mathrm{kPa}$ ). While the significant modification of physical properties allowed printing of complex structures, whether the increased stiffness influenced/stem cell differentiation and tissue formation was not examined. It has been shown that the osteogenic differentiation of HBMSCs within 3D matrices occurs preferentially in substrates from 10 to $30 \mathrm{kPa}$ [47], while vascularisation of tissue engineered constructs is favoured in softer matrices below $5 \mathrm{kPa}$ [48]. Furthermore, culture media and additional cell-signaling cues can produce a synergistic effect on cell function and differentiation $[48,49]$. In the bioink system reported herein, the addition of LPN resulted in unchanged mechanical strength of GeIMA ( $8 \mathrm{kPa})$ while maintaining significantly enhanced printability. This is significant given various studies have reported that the addition of LPN results in significant stiffening of matrices [31-33] which would render the hydrogel less permissive for vascular ingrowth. While the stiffness of our composite scaffolds is lower than the reported optimal stiffness for bone formation but higher than the optimal stiffness for vessel growth, our results suggest that the hydrogel was permissive for the multi-functional LPN filler to provide the required biofunctionality to achieve osteogenic differentiation and vascular ingrowth through growth factor localisation. Furthermore, the physical properties of LPN-GelMA hydrogels observed here is contrary to previously published studies, likely due to interaction of LPN with the novel visible light photoinitiating system employed. The current results indicate that the presence of LPN does interfere with the visible light mediated crosslinking reaction of the GeIMA macromere. This is evidenced by the increase in the soluble fraction and swelling ratio of the formulations examined. 
LPN has been shown to be able to interact with a plethora of molecules and materials as a consequence of the intrinsic dual-charge property of the nanoclay $[21,45,46,50]$. As previously reported, LPN strongly interacts with GeIMA at nanoclay concentrations equal to, or higher than, 1 wt \% [31]. The current studies indicate that while addition of LPN did not increase the overall stiffness of the gel, LPN reinforced the less dense hydrogel structure, reducing any significant loss in physical integrity, enhancing maintenance of shape fidelity in bioprinted scaffolds. A previous study demonstrated rheological characterisation and extrusion-printing of LPN blended with GeIMA [31]. However, while showing the benefit of LPN addition in rheological testing, a single formulation of LPN-GelMA was assessed for printing and no cells were used in the process, limiting the understanding of LPN inclusion influence on bioink printability and cell behaviour necessary for bioprinting of cell-laden bioinks. In all formulations examined in this current study, LPN addition increased bioink viscosity, which in turn translated to improved control over extruded filament quality. GelMA bioinks have narrow biofabrication window due to temperature sensitivity as discussed earlier [40]. Temperature variations can arise from multiple sources, such as the bioink reservoir, the nozzle, the building plate and the ambient temperature. In this study, we demonstrate that LPN inclusion in GelMA resulted in a robust bioink, with reduced sensitivity to temperature variations that enabled generation of scaffolds with high shape fidelity. It has been shown that pre-cooling GeIMA bioinks enhances viscosity and can improve printability, although the effects on fibre stacking, fusion, and interconnectivity of the scaffold are less clear [38]. Moreover, the possibility of damage to cells due to over-gelation of cooled bioinks, increasing shear stress, remains unclear. Another approach used a cold building plate at $5^{\circ} \mathrm{C}$ to fabricate GelMA constructs [46]. It is uncertain whether rapid temperature changes affect the viability and functionality of encapsulated stem cells. In the current study, addition of nanoclay helped to maintain hydrogel mechanical stability, increased viscosity and allowed for shear thinning behaviour at the printability window. Furthermore, nanocomposite bioinks could be 
extruded at $20.5^{\circ} \mathrm{C}$ and formed stable filaments that could be stacked through multiple layers, retaining porosity in all dimensions even post equilibrium swelling. In a clinical setting, such an optimised bioink offers significant potential as a cell carrier that could be used in combination with a biodegradable support thermoplastic or ceramic scaffold to provide a stable biofabricated implantable construct [51-53]. The high shape fidelity of the scaffolds would potentially promote blood vessel ingrowth, host integration, nutrient and oxygen diffusion and improved tissue formation while the support scaffold would address the considerable compressive stiffness and mechanical properties necessary for bone regeneration that clearly a LPN-GelMA construct alone would not support. The ability to bioprint LPN-GelMA constructs with candidate hybrid support scaffolds is currently the focus of ongoing work within our groups.

Pivotal for the achievement of the functionality of implantable constructs is the survival and proliferation of encapsulated living cells within scaffold matrices [54]. The biocompatibility of LPN-GelMA was investigated by the encapsulation of HBMSCs and long-term osteogenic culture and were found to preserve cell viability and proliferation for up to 21 days in vitro. We further demonstrated that bioprinted HBMSC-laden LPNGelMA bioinks were able to support osteogenic differentiation even in the absence of the osteoinductive drug dexamethasone, as shown by formation of mineralised nodules. While it has been previously shown that Laponite can act as an osteoinductive cue [55], this is the first time, to our knowledge, that this capacity has been investigated within a 3D bioprinted cell-laden LPN-GeIMA construct.

A major advantage of the LPN nanoparticles is their intrinsic capacity to spatially localise drugs on the surface of the charged nanodiscs [27]. Such an approach has been extensively studied for the in situ delivery of chemotherapeutics [56,57], wound healing $[58,59]$ and bone regeneration [29], Currently, bone defects are treated with absorbable collagen sponges (ACS) loaded with clinical dose of BMP-2. Recent studies have confirmed the efficacy of rhBMP-2 loaded ACS in bone formation in vivo [60]. However, 
collagen sponges rely on absorption of BMP-2 needing a significant concentration of drug to be loaded to counter loss during implantation [61]. In an alternative approach, Visser et al. [62] have modified the rhBMP-2 protein to increase its affinity for a collagen polymer. However, modification of the BMP-2 protein is not trivial and expensive. Using LPN, Gibbs et al. [29] used physiological doses of BMP-2 (40 ng) to demonstrate bone formation in vivo due to the ability of the nanoclay to retain and localise the drug in situ. Lysozyme and BSA were used in the current study as analogues for BMP-2 and VEGF as a control following previous drug release investigations [27]. Both LPN-GelMA and GelMA absorbed the majority of the lysozyme in solution $\left(10 \mu \mathrm{g} \mathrm{m} \mathrm{m}^{-1}\right)$ within 1 hour indicating limited influence of LPN in the direct absorption of lysozyme.

In contrast, the presence of LPN displayed clear differences on the effect of BSA absorption $\left(100 \mu \mathrm{g} \mathrm{ml}^{-1}\right)$. The double charged LPN surfaces can preferentially interact with the positively charged BSA protein, while the positive rim can attract negatively charged lysozyme. We hypothesise that due to the elevated negative charge distribution on the LPN nanoparticles surfaces [63], a strong interaction between positively charged BSA and negative face of the LPN is evident, resulting, as one would anticipate, preferential interaction of the LPN with the BSA [64]. Nevertheless, the difference in BSA released from LPN-GeIMA and GeIMA appeared not to be significant after $24 \mathrm{~h}$. In previous studies [1,27], Laponite inclusion provided a clear improvement in drug retention within the polymeric matrix. However, BSA has been reported to be entrapped within gelatin-based hydrogels after 24h [65], implicating bonding of BSA with the GelMA polymeric network.

LPN-GelMA functionality was confirmed in samples absorbed with VEGF and implanted in a chicken chorioallantoic membrane (CAM) model. Simulating a clinical setting, where a construct could be loaded with growth factors prior to implantation, VEGF was absorbed onto the fabricated scaffolds to deliver an angiogenic stimulant critical in the bone regenerative process $[66,67]$. LPN-GelMA with absorbed-VEGF showed excellent integration with the chorioallantoic membrane, with several afferent 
blood vessels. Blood clots were found in proximity of LPN-GeIMA with VEGF and drugfree control, suggesting strong angiogenic potential [68,69]. Particularly, LPN-GelMAVEGF resulted in significant penetration of membrane vessels compared to GelMAVEGF, suggesting a greater retention ability of VEGF within LPN-GeIMA polymeric structure [70]. Indeed, LPN-GFs combination significantly improved endothelial sprouting in vitro compared to free GFs in the media, but leaving unclear the effect of the nanoclay-mediate localisation in vivo [27].

While GelMA gels demonstrated integration with the chick membrane, vessel penetration was limited, evidenced by histological analysis with Goldner's Trichrome. The LPN-GeIMA nanocomposite bioinks displayed potent angiogenic potential as well as the ability to sequester the majority of VEGF, thereby stimulating penetration of blood vessels and integration with the CAM membrane. In contrast, GeIMA-VEGF hydrogels supported only a peripheral network of major vessels, with limited vessel penetration in the core of the discs due to the burst release of the angiogenic factor to the surrounding tissue, as modelled and shown in the BSA release kinetics. In order to thoroughly evaluate the effect of incorporating LPN into GelMA hydrogels on drug loading and release capability, a simple casted hydrogel disc model was employed in this study. Given the increasing evidence that scaffold architecture, topology, and interconnecting porosity/channels can influence the host response to the material [71], we envision that the drug and growth factor release profile of the LPN-GeIMA nanocomposite bioinks can be further controlled or tailored by varying the architecture of the 3D bioprinted scaffolds. Future work will investigate the effect of angiogenic factor localisation in combination with a bioprinted LPN-GeIMA implant ex vivo and in vivo with the aim to ultimately fabricate implantable constructs to stimulate bone formation in pre-clinical models. 


\section{Conclusions}

Nanoclay composite bioinks present ideal characteristics, including viscoelastic and cell encapsulating properties, for skeletal biofabrication applications. Laponite nanoclay was blended with GelMA to create a novel visible-light curing composite formulation with excellent shape retention post swelling, tuneable viscoelastic properties and the potential to spatially control bioactive molecule release. Nanoclay addition significantly enhanced the biofabrication window for successful extrusion-based bioprinting and shape fidelity. Visible-light crosslinking produced viable 3D cell laden bioprinted constructs that could be maintained in culture over 21 days in vitro. Laponite addition did not significantly affect the hydrogel stiffness while, importantly, facilitating cell proliferation and osteogenic differentiation, evidenced by matrix formation over 3 weeks in the absence of osteogenic factors (e.g. dexamethasone). Furthermore, ex vivo CAM model studies demonstrated that nanoclay composites containing VEGF could stimulate angiogenesis with excellent integration across the chick vascularised membrane. LPNGelMA scaffolds showed enhanced angiogenic potential with evidence of major blood vessel infiltration and membrane integration.

The current studies illustrate the unique potential of multi-functional (osteogenic and angiogenic) Laponite-GelMA composite bioinks that confer an improved biofabrication window, support cell viability, cell proliferation, growth factor localisation and functional activity. Laponite-GelMA bioinks offer new vistas for tissue fabrication with significant implications for hard and soft tissue regeneration. 


\section{Acknowledgements}

The authors would like to thank Ms. Julia Wells and Dr Janos Kanczler of the University of Southampton Bone and Joint group for their technical support. This study was supported by the Royal Society of New Zealand Rutherford Discovery Fellowship (RDF-UOO1204; TW), the European Union FP7-IRSES Mobility Grant 'skelGEN' (under grant agreement $n^{\circ} 318553 ;$ TW \& RO), the Health Research Council of New Zealand Emerging Researcher First Grant and Sir Charles Hercus Fellowship (HRC 15/483 \& HRC 19/135; KL). Funding to RO from the Biotechnology and Biological Sciences Research Council (BBSRC LO21071/ and BB/L00609X/1) and UK Regenerative Medicine Platform Hub Acellular Approaches for Therapeutic Delivery (MR/K026682/1) and Acellular / Smart Materials - 3D Architecture (MR/R015651/1) as well as University of Southampton is gratefully acknowledged. We acknowledge the many useful discussions with past and current members of the Bone and Joint Research Group in Southampton, UK. 


\section{References}

[1] Ahlfeld T, Cidonio G, Kilian D, Duin S, Akkineni A R, Dawson J I, Yang S, Lode A O R O C and G M 2017 Development of a clay based bioink for 3D cell printing for skeletal application Biofabrication 9

[2] Mekhileri N V, Lim K S, Brown G C J, Mutreja I, Schon B S, Hooper G J and Woodfield T B F 2018 Automated 3D bioassembly of micro-tissues for biofabrication of hybrid tissue engineered constructs Biofabrication 1024103

[3] Groll J, Boland T, Blunk T, Burdick J A, Cho D, Paul D, Derby B, Forgacs G, Li Q, Mironov V A and Moroni L 2016 Biofabrication : Reappraising the definition in an evolving field Biofabrication 013001 1-6

[4] Groll J, Burdick J A, Cho D-W, Derby B, Gelinsky M, Heilshorn S C, Jüngst T, Malda J, Mironov V A, Nakayama K, Ovsianikov A, Sun W, Takeuchi S, Yoo J J and Woodfield T B F 2018 A definition of bioinks and their distinction from biomaterial inks Biofabrication 11013001

[5] Paxton N, Smolan W, Böck T, Melchels F, Groll J and Jungst T 2017 Proposal to assess printability of bioinks for extrusion-based bioprinting and evaluation of rheological properties governing bioprintability Biofabrication 9044107

[6] Ribeiro A, Blokzijl M M, Levato R, Visser C W, Castilho M, Hennink W E, Vermonden T and Malda J 2017 Assessing bioink shape fidelity to aid material development in 3D bioprinting Biofabrication 10014102

[7] Nichol J W, Koshy S T, Bae H, Hwang C M, Yamanlar S and Khademhosseini A 2010 Cell-laden microengineered gelatin methacrylate hydrogels Biomaterials 31 5536-44

[8] Van Den Bulcke A I, Bogdanov B, De Rooze N, Schacht E H, Cornelissen M and Berghmans H 2000 Structural and Rheológical Properties of Methacrylamide Modified Gelatin Hydrogels Biomacromolecules 1 31-8

[9] Yue K, Trujillo-de Santiago G, Alvarez M M, Tamayol A, Annabi N and Khademhosseini A 2015 Synthesis, properties, and biomedical applications of gelatin methacryloyl (GelMA) hydrogels Biomaterials 73 254-71

[10] Sinha R P and Häder D-P 2002 UV-induced DNA damage and repair: a review Photochem. Photobiol. Sci.1 225-36

[11] Fedorovich NE, Oudshoorn M H, van Geemen D, Hennink W E, Alblas J and Dhert W J A 2009 The effect of photopolymerization on stem cells embedded in hydrogels Biomaterials 30 344-53

[12] Lim K S, Schon B S, Mekhileri N V, Brown G C J, Chia C M, Prabakar S, Hooper G J and Woodfield T B F 2016 New Visible-Light Photoinitiating System for Improved Print Fidelity in Gelatin-Based Bioinks ACS Biomater. Sci. Eng. 2 1752-62

[13] Lim K S, Levato R, Costa P F, Castilho M D, Alcala-Orozco C R, Dorenmalen K M A van, Melchels F P W, Gawlitta D, Hooper G J, Malda J and Woodfield T B F 2018 Bioresin for high resolution lithography-based biofabrication of complex cell-laden constructs Biofabrication 1034101

[14] Bertassoni L E, Cardoso J C, Manoharan V, Cristino A L, Bhise S. N, Araujo A. W, 
Zorlutuna P, Vrana N E, Ghaemmaghami A M, Dokmeci M R and Khademhosseini A 2014 Direct-write bioprinting of cell-laden methacrylated gelatin hydrogels Biofabrication 624105

[15] Colosi C, Shin S R, Manoharan V, Massa S, Costantini M, Barbetta A, Dokmeci M R, Dentini M and Khademhosseini A 2016 Microfluidic Bioprinting of Heterogeneous 3D Tissue Constructs Using Low-Viscosity Bioink Adv. Mater. 28 677-84

[16] Schuurman W, Levett P A, Pot M W, van Weeren P R, Dhert W J A, Hutmacher D W, Melchels F P W, Klein T J and Malda J 2013 Gelatin-Methacrylamide Hydrogels as Potential Biomaterials for Fabrication of Tissue-Engineered Cartilage Constructs Macromol. Biosci. 13 551-61

[17] Zhao X, Liu S, Yildirimer L, Zhao H, Ding R, Wang H, Cui W and Weitz D 2016 Injectable Stem Cell-Laden Photocrosslinkable Microspheres Fabricated Using Microfluidics for Rapid Generation of Osteogenic Tissue Constructs Adv. Funct. Mater. 26 2809-19

[18] Khetan S, Guvendiren M, Legant W R, Cohen D M, Chen C S and Burdick J A 2013 Degradation-mediated cellular traction directs stem cell fate in covalently crosslinked three-dimensional hydrogels Nat. Mater. 12 458-65

[19] Malda J, Visser J, Melchels F P, Jüngst T, Hennink W E, Dhert W J A, Groll J and Hutmacher D W 2013 25th Anniversary Article: Engineering Hydrogels for Biofabrication Adv. Mater. 25 5011-28

[20] Visser J, Peters B, Burger T J, Boomstra J, Dhert W J A, Melchels F P W and Malda $\mathrm{J} 2013$ Biofabrication of multi-material anatomically shaped tissue constructs Biofabrication 535007

[21] Nguyen L H, Annabi N, Nikkhah M, Bae H, Binan L, Park S, Kang Y, Yang Y and Khademhosseini A 2012 Vascularized Bone Tissue Engineering: Approaches for Potential Improvement Tissue Eng. Part B Rev. 18 363-82

[22] Shin S R, Shin C, Memic A, Shadmehr S, Miscuglio M, Jung H Y, Jung S M, Bae H, Khademhosseini A, Tang X and Dokmeci M R 2015 Aligned Carbon Nanotube-Based Flexible Gel Substrates for Engineering Biohybrid Tissue Actuators Adv. Funct. Mater. 25 4486-95

[23] Navaei A, Saini H, Christenson W, Sullivan R T, Ros R and Nikkhah M 2016 Gold nanorod-incorporated gelatin-based conductive hydrogels for engineering cardiac tissue constructs Acta Biomater. 41 133-46

[24] Carretero M I and Pozo M 2009 Clay and non-clay minerals in the pharmaceutical industry. Part I. Excipients and medical applications Appl. Clay Sci. 46 73-80

[25] Carretero M I and Pozo M 2010 Clay and non-clay minerals in the pharmaceutical and cosmetic industries Part II. Active ingredients Appl. Clay Sci. 47 171-81

[26] Dawson J I and Oreffo R O C 2013 Clay: New opportunities for tissue regeneration and biomaterial design Adv. Mater. 25 4069-86

[27] Dawson J I, Kanczler J M, Yang X B, Attard G S and Oreffo R O C 2011 Clay gels for the delivery of regenerative microenvironments Adv. Mater. 23 3304-8

[28] Ruiz-Hitzky E, Aranda P, Darder M and Rytwo G 2010 Hybrid materials based on clays for environmental and biomedical applications J. Mater. Chem. 20 9306-21 
[29] Gibbs D M R, Black C R M, Hulsart-Billstrom G, Shi P, Scarpa E, Oreffo R O C and Dawson J I 2016 Bone induction at physiological doses of BMP through localization by clay nanoparticle gels Biomaterials 99 16-23

[30] Carrow J K, Cross L M, Reese R W, Jaiswal M K, Gregory C A, Kaunas R, Singh I and Gaharwar A K 2018 Widespread changes in transcriptome profile of human mesenchymal stem cells induced by two-dimensional nanosilicates Proc. Natl. Acad. Sci. 201716164

[31] Xavier J R, Thakur T, Desai P, Jaiswal M K, Sears N, Cosgriff-Hernandez E, Kaunas $\mathrm{R}$ and Gaharwar A K 2015 Bioactive nanoengineered hydrogels for bone tissue engineering: A growth-factor-free approach ACS Nano 9 3109-18

[32] Paul A, Manoharan V, Krafft D, Assmann A, Uquillas J A, Shin S R, Hasan A, Hussain M A, Memic A, Gaharwar A K and Khademhosseini A 2016 Nanoengineered biomimetic hydrogels for guiding human stem cell osteogenesis in three dimensional microenvironments J. Mater. Chem. B 4 3544-54

[33] Chimene D, Peak C W, Gentry J L, Carrow J K, Cross L M, Mondragon E, Cardoso G B, Kaunas R and Gaharwar A K 2018 Nanoengineered lonic-Covalent Entanglement (NICE) Bioinks for 3D Bioprinting ACS Appl. Mater. Interfaces 10 9957-68

[34] Kawamoto T and Shimizu M 2000 A method for preparing 2- to 50-micron-thick freshfrozen sections of large samples and undecalcified hard tissues. Histochem. Cell Biol. $113331-9$

[35] Wang X, Ao Q, Tian X, Fan J, Tong H, Hou W and Bai S 2017 Gelatin-based hydrogels for organ 3D bioprinting Polymers (Basel). 9

[36] Klotz B J, Gawlitta D, Rosenberg A J W P, Malda J and Melchels F P W 2016 Gelatin-Methacryloyl Hydrogels: Towards Biofabrication-Based Tissue Repair Trends Biotechnol.

[37] Bertlein S, Brown G, Lim K S, Jungst T, Boeck T, Blunk T, Tessmar J, Hooper G J, Woodfield T B F and Groll J 2017 Thiol-Ene Clickable Gelatin: A Platform Bioink for Multiple 3D Biofabrication Technologies Adv. Mater. 29 1-6

[38] Liu W, Heinrich M A, Zhou Y, Akpek A, Hu N, Liu X, Guan X, Zhong Z, Jin X, Khademhosseini A and Zhang Y S 2017 Extrusion Bioprinting of Shear-Thinning Gelatin Methacryloyl Bioinks Adv. Healthc. Mater. 16014511601451

[39] Gao G, Schilling A F, Hubbell K, Yonezawa T, Truong D, Hong Y, Dai G and Cui X 2015 Improved properties of bone and cartilage tissue from 3D inkjet-bioprinted human mesenchymal stem cells by simultaneous deposition and photocrosslinking in PEG-GelMA Biotechnol. Lett. 37 2349-55

[40] Mouser V H M, Melchels F P W, Visser J, Dhert W J A, Gawlitta D and Malda J 2016 Yield stress determines bioprintability of hydrogels based on gelatin-methacryloyl and gellan gum for cartilage bioprinting Biofabrication 8 1-13

[41] Daly A C, Critchley S E, Rencsok E M and Kelly D J 2016 A comparison of different bioinks for 3D bioprinting of fi brocartilage and hyaline cartilage Biofabrication 8 1-10

[42] McBeth C, Lauer J, Ottersbach M, Campbell J, Sharon A and Sauer-Budge A F 2017 3D bioprinting of GelMA scaffolds triggers mineral deposition by primary human osteoblasts Biofabrication 9

[43] Daly A C, Pitacco P, Nulty J, Cunniffe G M and Kelly D J 2018 3D printed 
microchannel networks to direct vascularisation during endochondral bone repair Biomaterials 162 34-46

[44] Bertassoni L E, Cecconi M, Manoharan V, Nikkhah M, Hjortnaes J, Cristino A L, Barabaschi G, Demarchi D, Dokmeci M R, Yang Y and Khademhosseini A 2014 Hydrogel bioprinted microchannel networks for vascularization of tissue engineering constructs Lab Chip 14 2202-11

[45] Chen Y C, Lin R Z, Qi H, Yang Y, Bae H, Melero-Martin J M and Khademhosseini A 2012 Functional human vascular network generated in photocrosslinkable gelatin methacrylate hydrogels Adv. Funct. Mater. 22 2027-39

[46] Billiet T, Gevaert E, De Schryver T, Cornelissen M and Dubruel P 2014 The 3D printing of gelatin methacrylamide cell-laden tissue-engineered constructs with high cell viability Biomaterials 35 49-62

[47] Huebsch N, Arany P R, Mao A S, Shvartsman D, Ali O A, Bencherif S A, RiveraFeliciano J and Mooney D J 2010 Harnessing traction-mediated manipulation of the cell/matrix interface to control stem-cell fate Nat. Mater. 9518-26

[48] Klotz BJ, Lim KS, Chang YX, Soliman BG, Pennings 1 , Melchels FPW, Woodfield TBF, Rosenberg AJWP, Malda J G D 2018 Engineering of a complex bone tissue model with endothelialised channels and capillary-like networks Eur. Cells Mater.35: 335-349

[49] Tan S J, Fang J Y, Yang Z, Nimni M E and Han B 2014 The synergetic effect of hydrogel stiffness and growth factor on osteogenic differentiation Biomaterials 35 5294-306

[50] Sheikhi A, Afewerki S, Oklu R, Gaharwar A K and Khademhosseini A 2018 Effect of ionic strength on shear-thinning nanoclay-polymer composite hydrogels Biomater.

Sci. 6 2073-83

[51] Woodruff M A and Hutmacher D W 2010 The return of a forgotten polymerPolycaprolactone in the 21st century Prog. Polym. Sci. 35 1217-56

[52] Roohani-Esfahani S-I, Newman P and Zreiqat H 2016 Design and Fabrication of 3D printed Scaffolds with a Mechanical Strength Comparable to Cortical Bone to Repair Large Bone Defects Sci. Rep. 619468

[53] Jakus A E, Rutz A L, Jordan S W, Kannan A, Mitchell S M, Yun C, Koube K D, Yoo S C, Whiteley H E, Richter C-P, Galiano R D, Hsu W K, Stock S R, Hsu E L and Shah R N 2016 Hyperelastic "bone": A highly versatile, growth factor-free, osteoregenerative, scalable, and surgically friendly biomaterial Sci. Transl. Med. 8 358ra127-358ra127

[54] Slaughter B V., Khurshid S S, Fisher O Z, Khademhosseini A and Peppas N A 2009 Hydrogels in Regenerative Medicine Adv. Mater. 21 3307-29

[55] Gaharwar A K, Mihaila S M, Swami A, Patel A, Sant S, Reis R L, Marques A P, Gomes ME and Khademhosseini A 2013 Bioactive silicate nanoplatelets for osteogenic differentiation of human mesenchymal stem cells Adv. Mater. 25 3329-36

[56] Wang G, Maciel D, Wu Y, Rodrigues J, Shi X, Yuan Y, Liu C, Tomas H and Li Y 2014 Amphiphilic Polymer-Mediated Formation of Laponite-Based Nanohybrids with Robust Stability and pH Sensitivity for Anticancer Drug Delivery. ACS Appl. Mater. Interfaces 6 16687-95

[57] Zhou B, Wu B, Wang J, Qian Q, Wang J, Xu H, Yang S, Feng P, Chen W, Li Y, Jiang 
$\mathrm{J}$ and Han B 2018 Drug-mediation formation of nanohybrids for sequential therapeutic delivery in cancer cells Colloids Surfaces B Biointerfaces 163 284-90

[58] Ghadiri M, Chrzanowski W and Rohanizadeh R 2014 Antibiotic eluting clay mineral (Laponite ${ }^{\circledR}$ ) for wound healing application: an in vitro study J. Mater. Sci. Mater. Med. 25 2513-26

[59] Kiaee G, Mostafalu P, Samandari M and Sonkusale S 2018 A pH-Mediated Electronic Wound Dressing for Controlled Drug Delivery Adv. Healthc. Mater. 18003961800396

[60] Kim C H, Ju M H and Kim B J 2017 Comparison of recombinant human bone morphogenetic protein-2-infused absorbable collagen sponge, recombinant human bone morphogenetic protein-2-coated tricalcium phosphate, and platelet-rich fibrinmixed tricalcium phosphate for sinus augmentation in rabb J. Dent. Sci. 12 205-12

[61] Friess W, Uludag H, Foskett S, Biron R and Sargeant C 1999 Characterization of absorbable collagen sponges as recombinant human bone morphogenetic protein-2 carriers Int. J. Pharm. 185 51-60

[62] Visser R, Arrabal P M, Becerra J, Rinas U and Cifuentes M 2009 The effect of an rhBMP-2 absorbable collagen sponge-targeted system on bone formation in vivo Biomaterials 30 2032-7

[63] Mongondry P, Tassin J F and Nicolai T 2005 Revised state diagram of Laponite dispersions J. Colloid Interface Sci. 283 397-405

[64] Zhao X, Lang Q, Yildirimer L, Lin Z Y, Cui W, Annabi N, Ng K W, Dokmeci M R, Ghaemmaghami A M and Khademhosseini A 2016 Photocrosslinkable Gelatin Hydrogel for Epidermal Tissue Engineering Adv.Healthc. Mater. 5 108-18

[65] Slavevska-Raicki R, Glavas-Dodov M, Simonoska M, Goracinova K, Mladenovska K and Janevic E 2002 BSA - loaded gelatin microspheres: Comparative studies on biodegradation and drug release in presence of collagenase and trypsin Maced. Pharm. Bull. 48 9-14

[66] Kanczler J M and Oreffo R O C 2008 Osteogenesis and angiogenesis: The potential for engineering bone Eur. Cells Mater. 15 100-14

[67] Habibovic P, Woodfield T, de Groot $K$ and van Blitterswijk C 2006 Predictive value of in vitro and in vivo assays in bone and cartilage repair--what do they really tell us about the clinical performance? Adv. Exp. Med. Biol. 585 327-60

[68] Moreno-Jiménez I, Hulsart-Billstrom G, Lanham S A, Janeczek A A, Kontouli N, Kanczler J M, Evans N D and Oreffo R O 2016 The chorioallantoic membrane (CAM) assay for the study of human bone regeneration: a refinement animal model for tissue engineering Sci. Rep. 632168

[69] Ribatti D, Nico B, Vacca A and Presta M 2006 The gelatin sponge-chorioallantoic membrane assay Nat. Protoc. 1 85-91

[70] Bai Y, Bai L, Zhou J, Chen H and Zhang L 2018 Sequential delivery of VEGF, FGF-2 and PDGF from the polymeric system enhance HUVECs angiogenesis in vitro and CAM angiogenesis Cell. Immunol. 323 19-32

[71] Wray L S, Rnjak-Kovacina J, Mandal B B, Schmidt D F, Gil E S and Kaplan D L 2012 A silk-based scaffold platform with tunable architecture for engineering critically-sized tissue constructs Biomaterials 33 9214-24 